\title{
Optimum Equilibrium Passenger Flow Control Strategies with Delay Penalty Functions under Oversaturated Condition on Urban Rail Transit
}

\author{
Yonghao Yin $\mathbb{D}^{1},{ }^{1}$ Dewei Li $\mathbb{D},{ }^{2}$ Kai Zhao $\mathbb{D}^{3},{ }^{3}$ and Ruixia Yang $\mathbb{D}^{2}$ \\ ${ }^{1}$ Institute of Artificial Intelligence and Robotics (IAIR), Key Laboratory of Traffic Safety on Track of Ministry of Education, \\ School of Traffic and Transportation Engineering, Central South University, Changsha 410075, Hunan, China \\ ${ }^{2}$ State Key Lab of Rail Traffic Control and Safety, Beijing Jiaotong University, Beijing 100044, China \\ ${ }^{3}$ Railway and Station Design Research Department, China Railway Siyuan Survey and Design Group Co., Ltd., \\ Wuhan 430063, China \\ Correspondence should be addressed to Dewei Li; dwli.bjtu@163.com
}

Received 10 December 2019; Revised 3 December 2020; Accepted 6 February 2021; Published 25 February 2021

Academic Editor: Gonçalo Correia

Copyright (C) 2021 Yonghao Yin et al. This is an open access article distributed under the Creative Commons Attribution License, which permits unrestricted use, distribution, and reproduction in any medium, provided the original work is properly cited.

\begin{abstract}
When passengers are oversaturated in the urban rail transit system and a further increase of train frequency is impossible, passenger flow control strategy is an indispensable approach to avoid congestion and ensure safety. To make the best use of train capacity and reduce the passenger waiting time, coordinative flow control is necessary at each station on a line. In most published studies, the equilibrium of passenger distributions among different stations and periods is not considered. As a result, two issues occur making it hard to implement in practical. First, a large number of passengers are held up outside a small number of stations for very long time. Second, there is a large variation of controlled flows for successive time intervals. To alleviate this problem, a single-line equilibrium passenger flow control model is constructed, which minimizes the total passenger delay. By applying different forms of the delay penalty function (constant and linear), flow control strategies such as independent flow control and equilibrium flow control can be reproduced. An improved simulated annealing algorithm is proposed to solve the model. A numerical case is studied to analyze the sensitivity of the functions, and the best parameter relationship in different functions could be confirmed. A real-world case from Batong Line corridor in Beijing subway is used to test the applicability of the model and algorithm, and the result shows that the solution with linear delay penalty functions can not only reduce the total passenger delay but also equilibrate the number of flow control passengers on spatial and temporal.
\end{abstract}

\section{Introduction}

1.1. Background. With the development of urban rail transit, the number of passengers is increasing and the passenger demand has been far exceeded the transportation capacity of the railway system. The congestion has become one of the most severe problems which is affecting the operation safety and service level of urban rail transit in China. So far, there are two measures to dispose the congestion problem: (i) from the demand side, controlling the arrival passenger flow, such as using passenger flow control strategy at the entrance of the stations [1-3]; (ii) from the supply side, adjusting the train operations, such as adjusting train headway and stop plan [4-6].

However, the capacity of the oversaturated line almost reaches the upper bound during peak periods. The headway of trains is the minimum and all trains stop at every station in peak hours. It is almost impossible to improve the transport capacity of the line by increasing the train frequency of those busy lines. As a result, to ease the traffic pressure, the passenger flow control is one of the most effective and convenient ways.

In most of the urban rail transit systems of China, passengers have to pass the security check first, then swipe 
card to enter the station, walk through the passageway, go to the platform, wait for the arrival train, and board the train when the capacity of the train is sufficient. When the trains are out of capacity, passengers will be left behind at the platform while passengers continue coming (as shown in Figure 1(a)). In this situation, the additional waiting time for those passengers is inevitable, called the passenger left-behind delay. When the transit system is oversaturated, to avoid the congestion at the platform of the station, it is better for passengers to wait at the entrance than remaining at the platform. Considering the limited capacity of platforms and the reserved train capacity for other stations (to make full use of capacity), passengers should be controlled at the entrance before they pass the security check (as shown in Figure 1(b)), and the delay due to flow control at the entrance is called the passenger flow control delay.

The passenger flow control strategy determines the number of controlled passengers at the entrances of stations per period. They are forbidden to enter the station in this period. The flow control strategy compels passengers to wait at the entrance instead of waiting at the crowded platform. However, so far, the strategy of the passenger flow control still highly relies on the personal experience of the operators in current system. Furthermore, to make full use of train capacity, the cooperative flow control strategy at different stations and in different periods should be considered. Besides, to facilitate the implement of the strategy in realworld case, the equilibrium of passenger flow control strategy at successive stations and in successive periods should also be considered. Therefore, formulating quantitative for equilibrium passenger flow control strategy has a great significance.

1.2. Literature Review. Originally, the passenger flow control strategy was used on the field of bus transportation, namely, boarding limit strategy. Boarding limit strategy could be considered as a strategy that a fraction of the passengers who are waiting to board the bus might be requested to wait for the next bus even when the bus has capacity. The reason is that the request of subsequent stations might be more urgent or there are more passengers who are waiting at the following stations. The boarding limit strategy could change the temporal distribution of passenger demand, which could make full use of the vehicle capacity. Delgado et al. [7,8] developed an optimal model, which could execute two strategies: holding and boarding limits. Specifically, the boarding limits as a potentially attractive control mechanism could speed up the service. The application of these strategies allowed the buses to travel at a lower capacity, ensuring a better-balanced load and improving the comfort of the passengers. Ibarra-Rojas et al. [9] summarized that boarding limits strategy was one of the station control strategies in real-time control strategies, which could optimize the passenger waiting times efficiently. Sánchez-Martínez et al. [10] presented a model of flow control that explicitly considered the running time and dynamic demand, which outperformed the models in high demand cases.
Compared with the bus station, the situation on urban rail transit station is more complex because passengers should enter the station at the entrance, walk in the passageway, and wait for trains at the platform. It may not make sense to just control the number of boarding passengers, and the passengers should be controlled when they enter into the station. So, some researchers started to use the same method to optimize the quality of passenger service and passenger flow control strategy in the urban railway transit system. Baee et al. [11] proposed different boarding and alighting strategies to increase satisfaction level (which is represented by the number of passengers' collisions) and service success rate and reduce travel time in Tehran subway system. Li and Zhou [12] presented an improved algorithm about dynamic analysis of passenger flow (entering and exiting passengers at stations and transfer passengers between lines) to optimize the passenger organization in transfer station. In order to assess the effect of pedestrian traffic management on the boarding and alighting time at the metro station, simulation and experiments were conducted by Sebastián and Fernandez [13]. These studies focused on the passenger flow control of a single station, ignoring the influence of controlled stations on the corresponding downstream stations.

In recent years, some studies paid more attention on the coordinative flow control strategy for multiple stations on a line or in a network. He et al. [14] proposed a new coordinated-based passenger flow control method from a macroperspective in the subway network, which found out the bottleneck firstly based on the relationship of stations and links and then minimized the negative impact on irrelevant passengers to calculate the control strength and decide the controlled stations. From the microperspective, $\mathrm{Xu}$ et al. [2] took the impact of upstream strategy on downstream passengers into account and proposed a control strategy at the station. Considering the stop-skipping strategy and boarding limit, Jiang et al. [15] proposed a dynamic passenger flow control model and set the maximal passenger profit as the objective. Specifically, boarding limit and train operation organization were optimized simultaneously. Xu et al. [3] considered coordinative flow control of inbound and transfer passengers to simultaneously adjust the number of inbound and transfer passengers entering multiple stations or lines within a multistation coordinative passenger flow control model. A heuristic algorithm integrating genetic algorithm with the method of successive averages was used to dynamically control passenger crowding propagation. Jiang et al. [16] proposed a reinforcement learning-based simulation method to optimize the inflow volume with the aim of minimizing the passengers' safety risks. Then, Jiang et al. [17] considered the train rescheduling of skip-stopping and proposed a novel Q-learning-based simulation approach to coordinated optimize the passenger inflow control and train rescheduling with the objective of minimizing the penalty value of stranded passengers. With the objective function is to minimize passenger waiting time in the oversaturated line, Shi et al. [18] established an integer linear programming model to acquire a coordinative multistation passenger flow control strategy. Liu et al. [19] constructed a mixed integer 


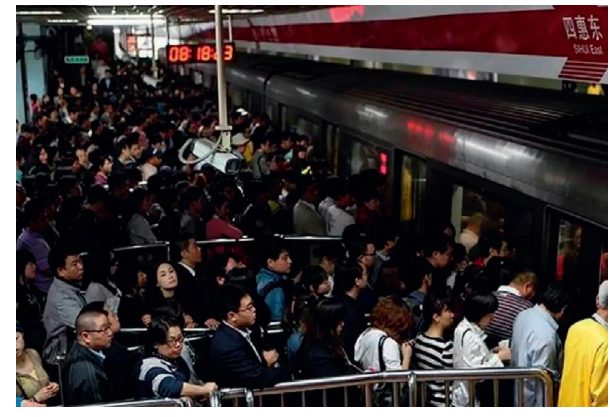

(a)

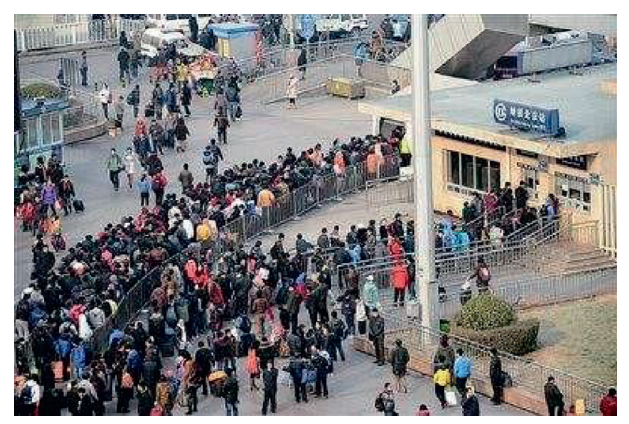

(b)

FIgURE 1. The left-behind and controlled passengers in different area. (a) Left-behind passengers at the platform. (b) Controlled passengers at the entrance.

linear programming model within the aim to improve train connectivity and reduce the number of stranded passengers, which is decomposed into two subproblems by the Lagrange theory and solved with CPLEX. Considering the passenger transfer behaviours, Shi et al. [20] established a multiobjective optimization to minimize passenger waiting time and minimize accumulation risk at the station. Yuan et al. [21] proposed a mixed integer linear programming model to minimize the passenger waiting time both outside the stations and on the platforms; the CPLEX software was used to solve the model and a real case of Beijing urban railway is tested. Considering the passengers on the station entrances and the station halls, Xue et al. [22] constructed an integer linear programming model to minimize the total passenger waiting time and keep the capacity of each key area of all stations is safety. Huang et al. [23] searched underutilized yet effective alternative routes and constructed an optimization model considering the penalties of in-vehicle congestion and transfers to alleviate peak hour congestion. Besides, there are also some scholars pay attention to the problem of coordinative flow control optimization in the real-time rescheduling $[23,24]$. The train dynamic rescheduling and passenger flow control are combined to restore the operation in order to achieve the fastest recovery of train operation and the least loss of passenger interests.

An overview of the aforementioned researches is listed in Table 1.

On one hand, about the spatial distribution, to our knowledge, some researches focus on the flow control strategy considering the coordination optimization between stations on a line or in a network, but the coordination optimization in most of the researches ignored the fairness of strategies. Specifically, coordination may be the most efficient strategy, but it may not be the most fairness strategy. The coordination strategy may serve more passengers or save more passengers waiting time (because of their target with minimum passenger waiting time), but the passengers at some stations with low flow may be served very late, which is not fair for them. Therefore, the fairness, such as equilibrium, of flow control strategy should be considered. On the other hand, most of the researches ignored the temporal distribution of strategy the equilibrium strategy between periods makes the flow control easy to apply. In practical application, the strategy with great fluctuation between each period is difficult for the operators to realize accurately and this has a directly effect on the flow control result. Moreover, the discussion of relationship between flow control passengers and left-behind passengers are rare in recent studies, which may have a great influence on flow control strategy.

1.3. Main Contributions. In this study, a flow control model is constructed for the line, in which the line is unidirectional or the line is bidirectional but the inflow with opposite directions can be controlled, respectively. The minimum total passenger delay is taken as the objective, which includes the flow control delay and the left-behind delay. Two forms of delay penalty functions, constant and linear, in objective function are proposed, and two models, nonequilibrium one and equilibrium one, are constructed, respectively. To ensure safety of passengers in rail transit system, the capacity of trains and the capacity of platforms are considered in the model. The model is solved by the improved simulated annealing (ISA) algorithm which is based on the random disturbance operator. A numerical study is used to test the models, and the results of ISA algorithm and CPLEX solver are compared to verify the efficiency of the algorithm. The sensitivity of these functions in two models is analyzed to find the optimal factors. Furthermore, a real-world case study, Batong Line of Beijing urban rail transit, is used to verify the reliability of the model and the algorithm, and the solution in two models with different functions is compared to find the equilibrium flow control strategy.

The contributions of this study are as follows:

(1) An equilibrium passenger flow control model was proposed by applying linear delay penalty factor in the model. Through equilibrium flow control, the number of passengers at different stations and in different flow control periods is collaboratively optimized, so that some situations which may lead to extensively long delays for a small number of stations were avoided. Besides, equilibrium passengers controlled in different periods can ease the flow control implementation in practice. The proposed model considered two forms of delay: flow control delay and retention delay, which are the main source of delay for passengers traveling in the urban rail transit system. 
TABLE 1: Overview of the existing flow control researches.

\begin{tabular}{|c|c|c|c|c|c|}
\hline Source & Objectives & Algorithm & $\mathrm{CC}$ & CES & CET \\
\hline Baee et al. [11] & Max. service success rate and min. travel time & Simulation & $\times$ & $x$ & $x$ \\
\hline $\begin{array}{l}\text { Sebastián and } \\
\text { Fernandez [13] }\end{array}$ & Min. passenger service time & Simulation & $x$ & $x$ & $x$ \\
\hline $\mathrm{Xu}$ et al. [2] & Min. average service time and max. served passengers & Simulation & $x$ & $x$ & $x$ \\
\hline Jiang et al. [17] & Min. penalty value of stranded passengers & Q-learning-based simulation & $\checkmark$ & $x$ & $x$ \\
\hline Li et al. [1] & Min. timetable error, headway deviation, and amplitude & $\begin{array}{l}\text { Model predictive control } \\
\text { algorithm }\end{array}$ & $\checkmark$ & $x$ & $\times$ \\
\hline Jiang et al. [15] & Max. profit of boarding and controlled passengers & Heuristic algorithm & $\checkmark$ & $x$ & $x$ \\
\hline Shi et al. [18] & Min. waiting time & Heuristic method and CPLEX & $\checkmark$ & $x$ & $x$ \\
\hline Liu et al. [19] & $\begin{array}{l}\text { Max. number of train services, min. number of passengers } \\
\text { waiting time, and min. amplitude of control }\end{array}$ & $\begin{array}{c}\text { Lagrangian relaxation algorithm } \\
\text { and CPLEX }\end{array}$ & $\checkmark$ & $x$ & $\checkmark$ \\
\hline $\mathrm{Xu}$ et al. [3] & Min. passenger travel time & Genetic algorithm & $\checkmark$ & $\checkmark$ & $\times$ \\
\hline Shi et al. [20] & Min. passenger waiting time and accumulation risks & CPLEX & $\checkmark$ & $x$ & $\checkmark$ \\
\hline Huang et al. [23] & Min. generalized time utility & Load balancing algorithm & $\checkmark$ & $\checkmark$ & $\times$ \\
\hline Hao et al. [24] & Min. operation costs and passenger loss & $\begin{array}{l}\text { Approximate dynamic } \\
\text { programming approach }\end{array}$ & $\checkmark$ & X & $\times$ \\
\hline This study & Min. total delay (flow control delay and left-behind delay) & $\begin{array}{l}\text { Improved simulated annealing } \\
\text { algorithm }\end{array}$ & $\checkmark$ & $\checkmark$ & $\checkmark$ \\
\hline
\end{tabular}

Notes: Min.: minimum; Max.: maximum; CC: consider coordination; CES: consider equilibrium on spatial distribution; CET: consider equilibrium on temporal distribution.

(2) The flow control strategy is controlled by delay penalty function. By applying different forms of the penalty functions, flow control strategies such as nonequilibrium flow control and equilibrium flow control can be reproduced. Moreover, by applying the value of penalty functions, we can control the number of passengers in different area of a station, i.e., at the platform or outside the entrance.

(3) An improved simulated annealing algorithm was constructed to solve the proposed models, which was based on the random disturbance operator. The algorithm could solve the model with high accuracy and high efficiency.

The remainder of this paper is structured as follows. In Section 2, the single-line passenger flow control problem is described. Section 3 introduces two flow control models with different forms of delay penalty functions: nonequilibrium one and equilibrium one. In Section 4, an improved simulated annealing algorithm which is based on random disturbance operator is proposed. In Section 5, a numerical study is used to analyze the sensitivity of parameters in the model and the best parameter relationship within different forms of functions was obtained. Section 6 uses the Batong Line in Beijing urban rail transit as a real-world case study to verify the effectiveness of the model and algorithm. Section 7 presents conclusions.

\section{Problem Statement}

2.1. Problem Definition. To guarantee the operation order of the trains and the safety of passengers, the passenger flow has to be guided or controlled by operators when the passengers are oversaturated in the urban rail transit system. In fact, there are various passenger flow control strategies; some of them may be the most efficient strategy (which means the total passenger delay is minimum), and some of them may be the fairest strategy (which means the strategy is equilibrium for every passenger). Every strategy has its advantages and disadvantages.

The passenger flow control at stations in one direction of a single railway line or a unidirectional railway line is considered. As shown in Figure 2, the station numbers in turn are $1,2,3, \ldots, S$, and trains serve from the first station to the last station. To simplify the issue, overtaking is not considered. Besides, all trains turn back at the terminal station. There are TE flow control periods and each period $t$ with the same length ( $\Delta t$ minute). The research time horizon is denoted as $\left[1, T_{\text {total }}\right]$, where $T_{\text {total }}$ equals to $\mathrm{TE} * \Delta t$. The passenger arrival rate $\mathrm{AR}_{s}(t)$ is given, which means the number of arriving passengers at the entrance of station $s$ during period $t$, which is so short that the passenger arrival rate is steady. The number of trains, train capacity, and operation parameters (including running times, dwell times, and headways, etc) are given.

The objective is to get the optimal flow control strategy that minimize the total passenger delay, which includes the flow control delay at the entrance and the left-behind delay at the platform. The flow control delay refers to the waiting time of the passengers outside the station caused by the flow control strategy; the left-behind delay refers to the waiting time of the passengers at the platform caused by the train without any remain capacity.

In this study, the equilibrium of flow control strategy is considered. The coordinated flow control strategy just considered the efficiency of the measure, which minimises the total passengers waiting time or delay. Different from coordination strategy, the equilibrium strategy may waste the efficiency, but it makes the boarding probability of passengers in different stations similar. The flow control strategy we proposed contains two aspects of equilibrium, the temporal and spatial equilibrium. The temporal equilibrium means that the number of 


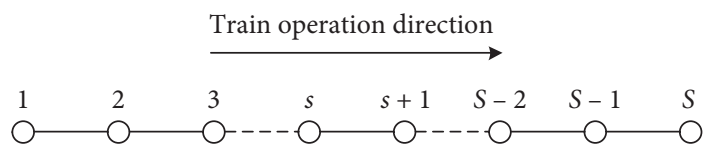

Figure 2: The representation of one direction on a single-line.

passengers entering each station varies smoothly with time, which is beneficial to the passenger flow management for the station and reduces the complexity of the arrival passenger flow organization. The spatial equilibrium refers to the number of controlled passengers on different stations are similar. These two spatial-temporal features will be reflected and explained by an illustrative case in Appendix C.

2.2. Notations. The indices, input parameters and variables in this study are listed and explained in Table 2.

2.3. Assumptions. To simplify the description of the equilibrium passenger flow control model, the following assumptions are introduced:

(1) Overtaking and crossing are prohibited at any position, and trains stop at each station in the line [25]. Running time, dwell time, and headway that the timetable includes are given. Therefore, trains run according to the timetable, regardless of the fluctuation in dwell time due to oversaturation.

(2) The passenger demand is time dependent, which is different from period to period. The time interval is predetermined, which is normally $2-5$ minutes. The passenger arrival ratio $\mathrm{AR}_{s}(t)$ at a station follows the uniform distribution in each time interval, because the fluctuation of passenger arrival ratio is small within few minutes in peak periods. This assumption is widely used in the literature [3]. Further more, passenger flow control strategy or the number of controlled passengers in each minute is also same in one interval.

(3) To simplify the expression of the model, the walking time of passengers in the passageway is assumed as a part of the waiting time, because the waiting time of passengers is difficult to obtain exactly and it is different from person to person.

(4) The passengers are assumed to board the first train with enough remaining capacity even though it is crowded [10], which avoids the waste of train capacity.

(5) The train dwell time is long enough for passengers to board and alight. The platform capacity for alight passengers to exit the stations is enough [3].

(6) Only one direction passenger inflow is considered in this study. Specifically, for the bidirectional line, the inflow with different directions can be controlled, respectively, by setting up the fencing facility at the halls [18].

\section{Single-line Equilibrium Flow Control Model}

3.1. Objectives. The total travel time includes the travel time on train, waiting time out of the train, and the access and egress times. Consider that the passenger delay usually occurs in the accessing process because of the oversaturated passenger flow, which is viewed as the passenger waiting time. To reduce the total travel time of passengers, the total waiting time of passengers out of the train should be discussed.

As shown in Figure 3, the total waiting time of passengers $\left(\mathrm{TW}_{\text {total }}\right)$ includes the flow control delay at the entrance $\left(\mathrm{TD}_{\text {control }}\right)$, passenger waiting time at the platform $\left(\mathrm{TW}_{\text {platform }}\right)$, and left-behind delay at the platform $\left(\mathrm{TD}_{\text {left-behind }}\right)$, which is expressed as equation (1). The flow control delay at the entrance is the delay of passengers who are controlled at the entrance, which is due to the flow control strategy in congestion periods. Because the passenger arrival rate follows the uniform distribution in each period (Assumption (2)), the first flow control delay is half duration of a flow control period. But, if the passengers are controlled more than once, the delay of them is underestimated, which lost a half duration for each second or multiple controlled passengers. To add the extra flow control delay for the second or multiple controlled passengers $\left(\widehat{q}_{s}^{\text {control }}(t)\right)$, an extra flow control delay should be included (as shown in equation (2)). The passenger waiting time at the platform refers to the waiting time at the platform before boarding. Because the passenger arrival rate on the platform also follows the uniform distribution, the waiting time of passengers is half of duration of the headway as shown in equation (3), which takes the walking time into account (Assumption (3)). The number of passengers who have gone into the station and the waiting time at the platform has notable positive correlation. In other words, if the number of entering passengers is fixed in study horizon, the waiting time on the platform is fixed. The left-behind delay is the delay of passengers who are left behind at the station due to the train capacity limitation, and they have to wait for the next train. Their delay should add an extra headway or even several headways (if the passenger was left behind more than once), which is shown in equation (4).

In summary, the total travel time is related to the flow control delay and the left-behind delay, which belong to the total passenger delay. Therefore, it is proposed as the objective function.

$$
\begin{aligned}
\mathrm{TW}_{\text {total }}= & \mathrm{TD}_{\text {control }}+\mathrm{TD}_{\text {left-behind }}+\mathrm{TW}_{\text {platform }}, \\
\mathrm{TD}_{\text {control }}= & \sum_{t=1}^{\mathrm{TE}} \sum_{s=1}^{S-1}\left\{\left[q_{s}^{\text {control }}(t)-\widehat{q}_{s}^{\text {control }}(t)\right] * \frac{\Delta t}{2}\right\} \\
& +\sum_{t=1}^{\mathrm{TE}} \sum_{s=1}^{S-1}\left[\hat{q}_{s}^{\text {control }}(t) * \Delta t\right] \\
= & \sum_{t=1}^{\mathrm{TE}} \sum_{s=1}^{S-1}\left[q_{s}^{\text {control }}(t)+\widehat{q}_{s}^{\text {control }}(t)\right] * \frac{\Delta t}{2},
\end{aligned}
$$


TABLE 2: Index, parameters, and variables.

\begin{tabular}{|c|c|}
\hline \multicolumn{2}{|c|}{ Index parameters } \\
\hline$o, s$ & Station indices, $o, s=1,2,3, \ldots, S$ \\
\hline$r$ & Train index, $r=1,2,3, \ldots, R$ \\
\hline$t$ & Time index, $t=1,2,3, \ldots$, TE \\
\hline \multicolumn{2}{|c|}{ Input parameters } \\
\hline$\Delta t$ & The duration of each passenger flow control period; unit: minute \\
\hline$T_{\text {total }}$ & The study time horizon; unit: minute \\
\hline $\mathrm{AR}_{s}(t)$ & The passenger arrival rate at station $s$ at period $t$; unit: pax/minute \\
\hline$P_{o, s}$ & The proportion of passengers from station $o$ to station $s$ in the total number of passengers boarding at station $o$; unit: - \\
\hline$f^{0, s}$ & Train departure headway; unit: minute \\
\hline $\mathrm{Cap}_{s}^{P}$ & The maximum capacity of the platform on station $s$; unit: pax \\
\hline$h_{1}(\cdot), h_{2}(\cdot)$ & Delay penalty functions; unit: - \\
\hline $\mathrm{Cap}^{T}$ & The maximum loading passengers on a train; unit: \\
\hline$L_{s, r}(t)$ & $\begin{array}{c}\text { Train location parameter (binary parameter). If train } r \text { is at the station } s \text { at the beginning of period } t \text {, it is } 1 \text {; otherwise, it is } 0 \text {; } \\
\text { unit: }-\end{array}$ \\
\hline
\end{tabular}

Variables

$Z$

$\mathrm{TD}_{\text {total }}$

$\mathrm{TD}_{\text {control }}$

$\mathrm{TD}_{\text {left-behind }}$

TW platform

$q e_{s}^{\text {arrive }}(t)$

$q e_{s}^{\text {wait }}(t)$

$q e_{s}^{\text {enter }}(t)$

$q p_{s}^{\text {wait }}(t)$

$q p_{s}^{\text {alight }}(t)$

$q p_{s}^{\text {board }}(t)$

$q t_{r}^{\text {in }}(t)$

$q t_{r}^{\text {capacity }}(t)$

$\overline{q s}^{\text {control }}(t)$

$\bar{q}^{\text {control }}(s, t)$

$\mathrm{SEI}_{t}$

SEI

TEI
$\mathrm{TW}_{\text {total }}$

$q p_{s}^{\text {left-behind }}(t)$

The value of the objective functions; unit: minute

Total delay of passenger travel time; unit: minute

Flow control delay of passengers at the entrance; unit: minute

Left-behind delay of passengers at the platform; unit: minute

Passenger waiting time at the platform; unit: minute

Total waiting time of passengers; unit: minute

The number of arriving passengers at the entrance of station $s$ at period $t$; unit: pax

The number of waiting passengers at the entrance of station $s$ at period $t$; unit: pax

The number of entering passengers at the entrance of station $s$ at period $t$; unit: pax

The number of waiting passengers at the platform of station $s$ at period $t$; unit: pax

The number of alighting passengers at station $s$ at period $t$; unit: pax

The number of boarding passengers at station $s$ at period $t$; unit: pax

The number of left-behind passengers at station $s$ at period $t$; unit: pax

The number of passengers in train $r$ at the end of period $t$; unit: pax

Remainder capacity of train $r$ at the end of period $t$; unit: pax

Average value of controlled passengers at all stations at period $t$; unit: -

Variation value of controlled passengers at station $s$ at period $t$; unit: -

Spatial equilibrium indicator at period $t$; unit: -

Spatial equilibrium indicator; unit: -

Temporal equilibrium indicator; unit: -

Decision variables

$q_{s}^{\text {control }}(t) \quad$ The number of controlled passengers by flow control strategy at the entrance of station $s$ at period $t$; unit: pax

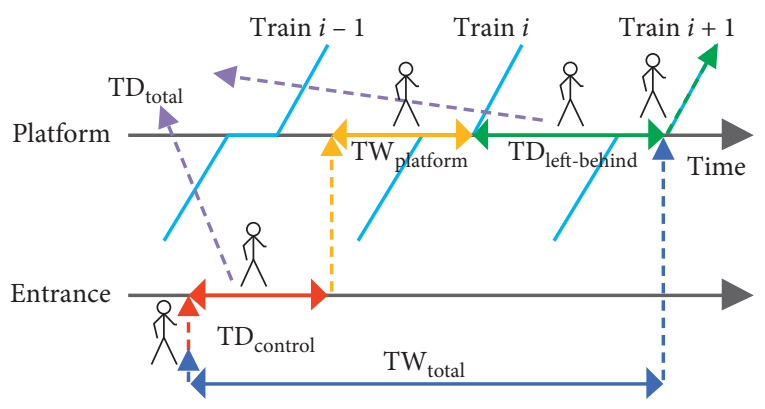

FIGURE 3: The relationship of delay and waiting time variables.

$$
\mathrm{TW}_{\text {platform }}=\sum_{t=1}^{\mathrm{TE}} \sum_{s=1}^{S-1}\left[q p_{s}^{\text {board }}(t) * \frac{f}{2}\right]
$$

$$
\mathrm{TD}_{\text {left-behind }}=\sum_{t=1}^{\mathrm{TE}} \sum_{s=1}^{S-1}\left[q p_{s}^{\text {left-behind }}(t) * f\right] .
$$

The goal of flow control strategy is to reduce the total passenger delay $\left(\mathrm{TD}_{\text {total }}\right)$ that is the sum of passengers' delay at all stations in study horizon. As shown in equation (5), the total passenger delay at each station consists of the flow control delay at the entrance $\left(\mathrm{TD}_{\text {control }}\right)$ and the left-behind delay at the platform $\left(\mathrm{TD}_{\text {left-behind }}\right)$. It is worth noting that the passenger flow strategy may lead to second or multiple retentions at the entrance. The objective function is to minimize weighted sum of the flow control delay and leftbehind delay functions which is shown in equation (6). The weight functions $h_{1}(\cdot)$ and $h_{2}(\cdot)$ called delay penalty functions can take different mathematical forms, which can produce different flow control strategies. We will discuss this effect in detail in Section 3.3: 


$$
\begin{aligned}
\mathrm{TD}_{\text {total }}= & \mathrm{TD}_{\text {control }}+\mathrm{TD}_{\text {left-behind }}, \\
\min Z= & h_{1}(\cdot) * \mathrm{TD}_{\text {control }}+h_{2}(\cdot) * \mathrm{TD}_{\text {left-behind }} \\
= & h_{1}(\cdot) * \sum_{t=1}^{\mathrm{TE}} \sum_{s=1}^{S-1}\left[q_{s}^{\text {control }}(t)+\widehat{q}_{s}^{\text {control }}(t)\right] * \frac{\Delta t}{2} \\
& +h_{2}(\cdot) * \sum_{t=1}^{\mathrm{TE}} \sum_{s=1}^{S-1}\left[q p_{s}^{\text {left-behind }}(t) * f\right] .
\end{aligned}
$$

$$
\begin{aligned}
& q e_{s}^{\text {arrive }}(t)=\mathrm{AR}_{s}(t) * \Delta t, \quad \text { for } \forall s=1,2, \ldots, S-1, \forall t=1,2, \ldots, \mathrm{TE}, \\
& q e_{s}^{\text {wait }}(t)=q e_{s}^{\text {arrive }}(t)+q_{s}^{\text {control }}(t-1), \quad \text { for } \forall s=1,2, \ldots, S-1, \forall t=2,3, \ldots, \mathrm{TE}, \\
& q e_{s}^{\text {enter }}(t)=q e_{s}^{\text {wait }}(t)-q_{s}^{\text {control }}(t), \quad \text { for } \forall s=1,2, \ldots, S-1, \forall t=1,2, \ldots, \mathrm{TE}, \\
& \widehat{q}_{s}^{\text {control }}(t)= \begin{cases}q_{s}^{\text {control }}(t)-q e_{s}^{\text {arrive }}(t), & q_{s}^{\text {control }}(t)>q e_{s}^{\text {arrive }}(t), \\
0, & q_{s}^{\text {control }}(t) \leq q e_{s}^{\text {arrive }}(t),\end{cases}
\end{aligned}
$$

Equation (7) shows the number of passengers arriving at entrance at period $t$, which is the product of the passenger arrival rate per unit time and the time interval. Equation (8) shows the number of passengers waiting at entrance at the end of period $t$, which consists of the passengers arriving at period $t$ and the passengers controlled at period $t-1$. Equation (9) shows the number of entering passengers at entrance at period $t$, which is equal to the waiting passengers at period $t$ subtracting the controlled passengers at period $t$. Equation (10) shows the number of second or multiple controlled passengers at entrance at period $t$. When the number of controlled passengers is more than the arrived passengers, the waiting passengers in previous period will be controlled again.

3.2.2. Constraints of Passenger Boarding and Alighting. If train capacity is enough, the passengers on the platform will board the train and they will alight when the train stops at their destination. So, the constraints of passenger boarding and alighting are shown in the following equations:

$$
\begin{aligned}
q p_{s}^{\text {wait }}(t)= & \sum_{r=1}^{R} L_{s, r}(t) * q p_{s}^{\text {left-behind }}(t)+\left[1-\sum_{r=1}^{R} L_{s, r}(t)\right] \\
& *\left[q p_{s}^{\text {wait }}(t-1)+q e_{s}^{\text {enter }}(t)\right] \\
& \quad \text { for } \forall s=1,2, \ldots, S-1, \forall t=2,3, \ldots, \mathrm{TE}
\end{aligned}
$$

$$
\begin{aligned}
q p_{s}^{\text {left-behind }}(t)= & \sum_{r=1}^{R} L_{s, r}(t) *\left[q p_{s}^{\text {wait }}(t-1)+q e_{s}^{\text {enter }}(t)\right. \\
& \left.-q p_{s}^{\text {board }}(t)\right] \\
& +\left[1-\sum_{r=1}^{R} L_{s, r}(t)\right] * q p_{s}^{\text {left-behind }}(t-1), \\
& \text { for } \forall s=1,2, \ldots, S-1, \forall t=2,3, \ldots, \mathrm{TE},
\end{aligned}
$$

$$
\begin{aligned}
q p_{s}^{\text {board }}(t)= & \sum_{r=1}^{R} L_{s, r}(t) * \min \left\{q p_{s}^{\text {wait }}(t-1)\right. \\
& \left.+q e_{s}^{\text {enter }}(t), q t_{r}^{\text {capacity }}(t-1)+q p_{s}^{\text {alight }}(t)\right\}, \\
& \quad \text { for } \forall s=1,2, \ldots, S-1, \forall t=2,3, \ldots, \mathrm{TE},
\end{aligned}
$$

$$
\begin{aligned}
q p_{s}^{\text {alight }}(t)= & \sum_{r=1}^{R} L_{s, r}(t) * \sum_{o=1}^{s-1}\left[q p_{o}^{\text {board }}(t) * P_{o, s}\right], \\
& \quad \text { for } \forall s=2,3, \ldots, S-1, \forall t=1,2, \ldots, \mathrm{TE},
\end{aligned}
$$

$q t_{r}^{\text {capacity }}(t)=\operatorname{Cap}^{T}-q t_{r}^{\text {in }}(t), \quad$ for $\forall r=1,2, \ldots, R$,

$$
\forall t=1,2, \ldots, \mathrm{TE} \text {, }
$$




$$
\begin{gathered}
q t_{r}^{\text {in }}(t)=q t_{r}^{\text {in }}(t-1)-q p_{s}^{\text {alight }}(t)+q p_{s}^{\text {board }}(t), \\
\text { for } \forall r=1,2, \ldots, R, \forall t=2,3, \ldots, \mathrm{TE},
\end{gathered}
$$

Equation (11) shows the number of waiting passenger at the platform at the end of period $t$. If the train arrived at period $t$, it equals to the left-behind passengers at period $t$; otherwise, it equals to the waiting passengers at period $t-1$ and the entered passengers at period $t$. Equation (12) shows the number of left-behind passenger at the platform at the end of period $t$. If the train arrived at period $t$, it equals to the current waiting passengers (waiting passengers at period $t-1$ and entered passengers at period $t$ ) which excluded the boarded passengers at period $t$; otherwise, it equals to the left-behind passengers at period $t-1$. Equation (13) means the number of boarding passengers at the end of period $t$. If the train arrived at period $t$, it equals to the minimal between the current waiting passengers at the platform and the remain capacity on the train; otherwise, it equals to 0. Equation (14) indicates the number of alighting passengers at the station $s$ at the end of period $t$. If the train arrived at period $t$, it is the product of the passenger alighting rate and the number of passengers boarding the train; otherwise, it equals to 0. Equation (15) shows the number of residual loading capacity of train $r$ at the end of period $t$, which is equal to the train capacity minus the passengers on the train. Equation (16) shows the number of passengers on train $r$ at the end of period $t$, which is equal to the number of passengers on the train at former period and current boarding passengers minus current the alighting passengers. Equation (17) shows that the number of controlled passengers cannot go beyond the number of waiting passengers at the entrance. Equation (18) shows that the number of boarding passengers at the platform cannot exceed the number of waiting passengers, which is equal the number of waiting passengers on the platform at former period and the current entering passengers.

Equation (14) discussed the relationship of passengers between different stations; there exists a time offset of parameters between different stations, due to the running time and the waiting time of the train. Specifically, to eliminate the time offset of variables between the stations, the time shaft of the stations (except the first station) is moved before the calculation. The value of moving is the sum of the running time and the waiting time between the station and the first station, which is the time offset between the two stations. Based on the preprocess of the variables, the board passengers at the previous stations and the alight passengers at current station are in the same period $t$ for train $r$.
3.2.3. Constraints of Capacity. To guarantee the safety of passengers, the number of passengers at the platform and on the train should be restricted. Specifically, as shown in equation (19), the number of passengers on each train should not exceed the train capacity; as shown in equation (20), the number of passengers at platform should not be more than the platform capacity:

$$
q p_{s}^{\text {wait }}(t) \leq \operatorname{Cap}_{s}^{P}, \quad \text { for } \forall s=1,2, \ldots, S-1, \forall t=1,2, \ldots, \mathrm{TE},
$$

$$
q t_{r}^{\text {in }}(t) \leq \mathrm{Cap}^{T}, \quad \text { for } \forall r=1,2, \ldots, R, \forall t=1,2, \ldots, \mathrm{TE} .
$$

3.2.4. Variable Range Constraints. The range of decision variables is a natural number, and the range of intermediate variables is also natural number, which is shown in equations (21) and (22), respectively:

$$
q_{s}^{\text {control }}(t) \in N, \quad \text { for } \forall s=1,2, \ldots, S, \forall t=1,2, \ldots, \mathrm{TE},
$$

$$
\begin{aligned}
& q e_{s}^{\text {wait }}(t), q e_{s}^{\text {enter }}(t), \widehat{q}_{s}^{\text {control }}(t), q p_{s}^{\text {wait }}(t), q p_{s}^{\text {board }}(t), \\
& q p_{s}^{\text {lft }}(t), q p_{s}^{\text {alight }}(t), q t_{s}^{\text {in }}(t), q t_{s}^{\text {cap }}(t) \in N, \\
& \quad \text { for } \forall s=1,2, \ldots, S, \forall t=1,2, \ldots, \text { TE. }
\end{aligned}
$$

3.3. Delay Penalty Functions. The delay penalty functions $h_{1}(\cdot)$ and $h_{2}(\cdot)$ of two delay variables in the objective function determine how the delay is weighted, which dominates the flow control strategy. Different forms of delay penalty functions can produce different flow control strategies. Two types of delay penalty functions are discussed and two models with different objective functions are constructed, which are shown as follows:

Model I: nonequilibrium flow control model: in Model I, the delay penalty functions are set as constant type. The delay penalty functions are $h_{1}(\cdot)=\alpha_{1}$ and $h_{2}(\cdot)=\beta_{1}$, in which $\alpha_{1}$ and $\beta_{1}$ are parameters. The objective function is shown in equation (23) and follows the constraints (7)-(22). Constant delay penalty functions can directly show the influence of two forms of delays on passengers. The two forms of delay have a linear relationship with the number of delay passengers, and two forms of delay happen at different places. Specifically, the flow control delay happens at the entrance, and the left-behind delay happens at the platform. Therefore, the functions reflect the passenger perception of delay time at different facilities, namely, entrance and platform. The facility with delay penalty function of large value may reduce the number of waiting passengers there, and the place with delay penalty function of low value can reduce passenger antipathy to waiting. In other words, the value of functions can decide the place where the operators 
prefer passengers to wait. Therefore, the flow control delay and the left-behind delay can be balanced well by the constant delay penalty functions:

$$
\begin{aligned}
\min Z= & \alpha_{1} * \mathrm{TD}_{\text {control }}+\beta_{1} * \mathrm{TD}_{\text {left-behind }} \\
= & \alpha_{1} * \sum_{t=1}^{\mathrm{TE}} \sum_{s=1}^{S-1}\left[q_{s}^{\text {control }}(t)+\widehat{q}_{s}^{\text {control }}(t)\right] * \frac{\Delta t}{2} \\
& +\beta_{1} * \sum_{t=1}^{\mathrm{TE}} \sum_{s=1}^{S-1}\left[q p_{s}^{\text {left-behind }}(t) * f\right] .
\end{aligned}
$$

Obviously, the number of delay passengers at different stations in different periods is not considered by the constant delay penalty functions. Therefore, such a flow control strategy is a nonequilibrium solution. In other words, the flow control rate among successive stations or successive flow control periods can be unbalanced. As a result, the linear delay penalty functions are proposed and they will be discussed in Model II.

Model II: equilibrium flow control model: in Model II, the delay penalty functions are set as two linear functions, $h_{1}(\cdot)=\alpha_{2} * \mathrm{TD}_{\text {control }}$ and $h_{2}(\cdot)=\beta_{2} * \mathrm{TD}_{\text {left-behind }}$, respectively, in which $\alpha_{2}$ and $\beta_{2}$ are parameters. The objective function is shown in equation (24) and follows the constraints (7)-(22):

$$
\begin{aligned}
\min Z= & \alpha_{2} *\left(\mathrm{TD}_{\text {control }}\right)^{2}+\beta_{2} *\left(\mathrm{TD}_{\text {left- behind }}\right)^{2} \\
= & \alpha_{2} * \sum_{t=1}^{\mathrm{TE}} \sum_{s=1}^{S-1}\left\{\left[q_{s}^{\text {control }}(t)+\widehat{q}_{s}^{\mathrm{control}}(t)\right] * \frac{\Delta t}{2}\right\}^{2} \\
& +\beta_{2} * \sum_{t=1}^{\mathrm{TE}} \sum_{s=1}^{S-1}\left[q p_{s}^{\text {left-behind }}(t) * f\right]^{2}
\end{aligned}
$$

The above delay penalty functions have a quadratic relationship with the number of delayed passengers. Compared to constant delay penalty, the linear delay penalty with quadratic objective function in Model II can lead to an equilibrium solution for both time and space; the proof is shown in Appendix A.

3.4. Analysis of Model Complexity. Model I and Model II are both integer nonlinear programming models. They both have the same variables and constraints. The complexity of the models are determined by three preset parameters $S, R$, and TE on the considered line corridor, which are the number of stations, the number of trains, and the number of flow control periods, respectively. Table 3 lists the maximum number of variables and constraints. To illustrate the problem, an example with 10 stations and 10 trains in 10 periods is provided. There are 100 integer decision variables, 1,800 intermediate variables, and 1,136 constraints at most. With the prespecified parameters increasing, the number of variables and constraints will show a geometric increase. Furthermore, because the decision variable refers to the number of controlled passengers with high precision, the high-precision result is difficult to realize in reality. A nearoptimal solution is also acceptable as long as the solution is close to the global optimal solution and achieves satisfactory. It is also important to develop an easy methodology which can provide a good solution within acceptable computation time. Therefore, we develop a heuristic algorithm, namely, ISA algorithm, to obtain a feasible solution for the passenger flow control problem.

3.5. Performance Indicator of Equilibrium. To analyse the advantages and disadvantages of optimized flow control strategy, expect for the total passenger delay (including passenger left-behind delay and flow control delay), more performance indicators should be considered. Equilibrium is a flow control characteristic that is difficult to describe quantitatively. To discuss the temporal and spatial traits of passenger flow control strategy, spatial equilibrium indicator (SEI) and temporal equilibrium indicator (TEI) are proposed, which reflect the equilibrium of strategy.

3.5.1. Spatial Equilibrium Indicator. Spatial equilibrium indicator (SEI) is used to describe the deviation of passengers who enter different stations in the same period. The construction of the performance indicators involves the standard deviation in statistics. The average controlled passengers at different stations in the same period $\left(\overline{q s}^{\text {control }}(t)\right)$ are calculated by equation (25). The spatial equilibrium indicator in each period $\left(\mathrm{SEI}_{t}\right)$ is shown in equation (26). Then, the spatial equilibrium indicator is derived as shown in equation (27):

$$
\begin{gathered}
\overline{q s}^{\text {control }}(t)=\sum_{s=1}^{S-1} \frac{q_{s}^{\text {control }}(t)}{(S-1)}, \quad \text { for } \forall t=1,2, \ldots, \mathrm{TE}, \\
\mathrm{SEI}_{t}=\sqrt{\sum_{s=1}^{S-1} \frac{\left[q_{s}^{\text {control }}(t)-\overline{q s}^{\text {control }}(t)\right]^{2}}{(S-1)}}, \quad \text { for } \forall t=1,2, \ldots, \mathrm{TE},
\end{gathered}
$$

$$
\mathrm{SEI}=\sum_{t=1}^{\mathrm{TE}} \frac{\mathrm{SEI}_{t}}{(\mathrm{TE})}
$$

3.5.2. Temporal Equilibrium Indicator. Temporal equilibrium indicator (TEI) implies the fluctuation of passengers who enter the same station in different periods. The variation of controlled passengers $\left(\bar{q}^{\text {control }}(s, t)\right)$ can be shown as the gap between the average number of controlled passengers in former and latter periods and the number of controlled passengers in present periods (equation (28)). Then, the temporal equilibrium indicator is derived as shown in equation (29): 
TABLE 3: Scale of variables and constraints in the models.

\begin{tabular}{lr}
\hline Variables or constraints & Maximum total number \\
\hline Decision variable $q_{s}^{\text {control }}(t)$ & $S * \mathrm{TE}$ \\
Intermediate variables $q e_{s}^{\text {arrive }}(t), q e_{s}^{\text {wait }}(t), q e_{s}^{\text {enter }}(t), q p_{s}^{\text {wait }}(t), q p_{s}^{\text {alight }}(t), q p_{s}^{\text {board }}(t), q p_{s}^{\text {left-behind }}(t)$ & $7 * S * \mathrm{TE}$ \\
Intermediate variables $q t_{r}^{\text {in }}(t), q t_{r}^{\text {capacity }}(t)$ & $2 * R * \mathrm{TE}$ \\
Constraints of passengers at the entrance $(7)-(10)$ & $(S-1) *(4 \mathrm{TE}-2)$ \\
Constraints of passengers' boarding and alighting $(11)-(18)$ & $(S-1) *(6 \mathrm{TE}-5)+R(2 \mathrm{TE}-1)$ \\
Constraints of capacity (19) and $(20)$ & $(S+R-1) * \mathrm{TE}$ \\
\hline
\end{tabular}

$$
\begin{aligned}
\bar{q}^{\text {control }}(s, t)= & \left|\frac{\left[q_{s}^{\text {control }}(t+1)+q_{s}^{\text {control }}(t-1)\right]}{2-q_{s}^{\text {control }}(t)}\right| \\
& \text { for } \forall s=1,2, \ldots, S-1, \forall t=2,3, \ldots, \mathrm{TE}-1
\end{aligned}
$$

$$
\mathrm{TEI}=\sum_{s=1}^{S-1} \sum_{t=2}^{\mathrm{TE}-1} \bar{q}^{\text {control }} \frac{(s, t)}{[(S-1)(\mathrm{TE}-2)]}
$$

The two indicators are smaller, and the flow control strategy has more equilibrium, which means the deviation of controlled passengers is smaller at different stations and the fluctuation of that is smoother in different periods.

\section{Improved Simulated Annealing Algorithm}

In the proposed model, the decision variables are the number of controlled passengers on each station at each period, which is a series of integers with a finite range (the lower bound is 0 and the upper bound is the number of total arrival passengers), and the feasible solutions may be close and even similar. An algorithm is needed to randomly search the feasible solutions in a local area and also avoid falling into the local optimal solution. Therefore, simulated annealing (SA) algorithm is suitable, which is a random local search method with different search strategies, acceptance, and stop conditions, which is able to avoid falling into poor quality local optimal solution by accept nonimproved solution with certain probabilities. To guarantee the convergence of the algorithm, the accept probability is related to the temperature, with temperature cooling down and the acceptable probability goes to 0 [26]. SA originated from statistical mechanics, which is a solution method that is based on the physical process of simulated annealing. It was firstly proposed to use in combinatorial optimization problem by Kirkpatrick et al. [27]. The method has strong applicability and can obtain solutions arbitrarily close to the optimum [28].

4.1. The Framework of the ISA Algorithm. In flow control problem, the decision variable is the number of controlled passengers and the intermediate variables are also the number of passengers at each area, so the optimal result is difficult to find in large passenger flow. A number of passengers at some place have relationships with each other, a decrease in one place will lead to an increase in the other, so the range of search in each iteration is difficult to decide. For example, when the waiting passengers at the entrance entered station, the number of waiting passengers decreased and the number of entered passengers increased, and the amount of change is the same. To adapt to the flow control problem, an improved simulated annealing (ISA) algorithm with the random disturbance operator is constructed. After the algorithm introduction, the parameters will be explained in Table 4.

The pseudocode of the ISA algorithm is shown in Algorithm 1. The passenger demand, basic operation constraints, and basic algorithm parameters are set as the input datasets. Firstly, the algorithm will be initialized in Step 1, a solution without any flow control strategy will be given as the initial solution, and the temperature is set as the start temperature. Secondly, the current solution will be applied a random disturbance, and a new solution is produced in Step 2. Thirdly, the new value of objective functions will be calculated in Step 3 by equations (7)-(22). Then, in Step 4, the new solution will be accepted as the best solution if it is better than the current one, or the random bad solution accepted condition is existence. The temperature will be cooled down with the cooling rate, if the temperature is higher than the end temperature, and if not, the algorithm will be stopped. Finally, the best flow control strategy and the best objective value will be put out.

4.2. The Steps of the ISA Algorithm. Specifically, there are four steps. Step 1: initialization, Step 2: random disturbance, Step 3: result calculation, and Step 4: result acceptance in the ISA algorithm. The specific function of four steps is shown as follows:

\section{Step 1: initialization}

Before we calculate the flow control strategy, the value of parameters and variables should be initialized. Initialization includes algorithm initialization and model initialization. The algorithm initialization sets the start temperature Temp $S$, cooling rate $\Delta$ Temp, and end temperature Temp $E$. The model initialization sets the passenger arrival rate $\mathrm{AR}_{s}(t)$, the proportion of passengers at each station $P_{o, s}$, initial flow control strategy $X_{\text {init }}$, and initial result $E_{\text {init }}$. Specifically, the initial flow control strategy does not control any passengers; in other words, $q_{s}^{\text {control }}(t)$ is equal to 0 . The initial result can be calculated by Step 3, which will be detailed introduced as follows:

Step 2: random disturbance 
TABLE 4: Explanation of parameters in the ISA algorithm.

\begin{tabular}{lc}
\hline Parameters & Explanation \\
\hline$i$ & The index of iterations \\
Temp $(i)$ & The temperature at iteration $i$ \\
Temp $S$ & The start temperature \\
$\Delta$ Temp & The cooling rate \\
Temp $E$ & The end temperature \\
$X_{\text {init }}$ & The initial flow control strategy, which consists of $q_{s}^{\text {control }}(t)$ \\
$E_{\text {init }}$ & The initial result which is the value of objective function \\
$X_{\text {new }}$ & The flow control strategy in new solution \\
$E_{\text {new }}$ & The value of objective function in new solution \\
$X$ & The flow control strategy in current solution \\
$E$ & The value of objective function in current solution \\
$X_{\text {best }}$ & The best flow control strategy \\
$E_{\text {best }}$ & The value of objective function in best solution \\
Rand & A random parameter from 0 to 1 \\
MSol & The maximum order of magnitude of inboard passengers \\
$\rho$ & The precision of disturbance \\
$\Delta q_{s}^{\text {control }}(t)$ & The random disturbance operator for the number of controlled passengers at station $s$ at period $t$ \\
\hline
\end{tabular}

Input:

Passenger demand (passenger arrival rate, proportion of passengers);

Basic operation constraints (time horizon, running time, headway, train capacity, and platform capacity);

Basic algorithm parameters (start temperature, end temperature, cool rate, and disturbance precision).

Output:

The number of controlled passengers at the entrance at each station in each period $X_{\text {best }}$;

Value of objective function $E_{\text {best }}$.

Initialize: Step 1 (Initialization)

$X_{\text {best }} \longleftarrow X \longleftarrow X_{\text {init, }}, E_{\text {best }} \longleftarrow E \longleftarrow E_{\text {init, }}, \operatorname{Temp}(i) \longleftarrow$ Temp $S$

While $(\operatorname{Temp}(i)>$ Temp $E)$

Step 2 (Random disturbance) is used to produce a new solution: $X_{\text {new }}$;

Step 3 (Result computation) is used to compute the result of the new solution: $E_{\text {new; }}$;

Step 4 (Result selection) is used to select a result: $X$ and $E$;

$\operatorname{Temp}(i+1) \longrightarrow \operatorname{Temp}(i)-\Delta \mathrm{Temp}, i \longleftarrow i+1$

end While

Return $X_{\text {best }}, E_{\text {best }}$.

Algorithm 1: Improved simulated annealing algorithm.

With the temperature decreasing, the new solution will be obtained by random disturbance. Roulette wheel selection is used to produce a random disturbance operator $\Delta q_{s}^{\text {control }}(t)$. The random disturbance operator changes the strategy by $\Delta q_{s}^{\text {control }}(t)$, and then a new passenger flow control strategy $X_{\text {new }}$ can be obtained. Specifically, it will change the number of controlled passengers at each station in each period. To increase randomness, a random parameter Rand is set to note a random number from 0 to 1 . To control the disturbance in a reasonable range, the order of magnitude of the number of waiting passengers at the entrance MSol will be calculated at first (equation (30)). Equation (30) calculates the maximum order of magnitude of waiting passengers at the entrance.
Then, the disturbance is calculated by the equation (31). Finally, combine the current strategy with the random disturbance to get a new passenger flow control strategy $X_{\text {new }}$. Specifically, the step length of search is decided by the maximum order of magnitude MSol and the precision of disturbance $\rho$, which can be adjusted by the number of waiting passengers at the entrance and improve the efficiency of the algorithm. For example, the disturbance precision is 0.1 and Rand is 0.3 . If the maximum number of waiting passenger is 300 at period $t$, the magnitude is 100 and the random disturbance operator is 10 . On the other hand, if the maximum waiting passenger is 20 at period $t+1$, the magnitude is 10 and the random disturbance operator is 1: 


$$
\text { MSol }=10 \wedge\left\lfloor\log _{10} \operatorname{Max}\left\{q e_{s}^{\text {wait }} t \mid s=1,2, \ldots, S\right\}\right\rfloor
$$

$$
\Delta q_{s}^{\text {control }} t=\left\{\begin{array}{l}
\operatorname{Max}\{2,2 * \operatorname{MSol} * \rho\}, \quad \text { Rand } \in 0,0.25, \\
\operatorname{Max}\{1, \mathrm{MSol} * \rho\}, \quad \text { Rand } \in[0.25,0.5, \\
\operatorname{Min}\{-1,-\operatorname{MSol} * \rho\}, \quad \text { Rand } \in[0.5,0.75 \\
\operatorname{Min}\{-2,-2 * \operatorname{MSol} * \rho\}, \quad \text { Rand } \in[0.75,1
\end{array}\right.
$$

Step 3: result calculation

Because the number of passengers at every place have relationships with each other, the intermediate variables such as $q e_{s}^{\text {arrive }}(t), q e_{s}^{\text {wait }}(t), q e_{s}^{\text {enter }}(t), q p_{s}^{\text {wait }}(t)$, $q p_{s}^{\text {alight }}(t), q p_{s}^{\text {board }}(t)$, and $q p_{s}^{\text {left-behind }}(t)$ should be calculated at first. Based on the constraints (7)-(20), the intermediate variables can be calculated, if a passenger flow control strategy is given. The new passenger flow control strategy $X_{\text {new }}$ that includes the controlled passengers at each station in each period $q_{s}^{\text {control }}(t)$ is obtained by step 2 (random disturbance), so the intermediate variables in new solution can be calculated and the objective value in new solution $E_{\text {new }}$ can be calculated.

\section{Step 4:result acceptance}

Generally, the new solution will be accepted if the objective value in new solution $\left(E_{\text {new }}\right)$ is smaller than the current one $(E)$; otherwise, we retain the current solution. To avoid falling into a local optimal solution in the computation process, the nonimproved solution (with nonimproved objective value) will be accepted with a certain probability. The nonimproved solution will be accepted if the random nonimproved solution accepted condition (Rand $<\operatorname{Exp}\left[-\left(E_{\text {new }}-E\right) /\right.$ Temp (i)]) exists.

\section{Numerical Research}

At first, we constructed a small-scale numerical experiment to validate the effectiveness of the proposed single-line synchronous flow control model. The Model I was solved by exact solver CPLEX as well as the ISA algorithm; the result and the computation time will be compared. Then, the Model II was solved by the ISA algorithm, the results of Model I and Model II with different parameters are compared, and the sensitivity of delay penalty functions are analyzed.

5.1. Introduction of Numerical Case. In the numerical experiment, a line within one direction includes 5 stations and 4 sections. The study time horizon is 65 minutes (7:00-8:05). There are 5 trains, and all of them stop at each station. The running time of each train between two adjacent stations are $11,7,9$, and 12 minutes, respectively; the dwell time at each station is 2 minutes. As shown in Figure 4, trains run from station 1 to station 5 , and the headway of all the successive trains is 3 minutes. The first train departs from the first station at 7:05, from which the train location parameter $L_{s, r}(t)$ can be obtained. There are five flow control periods, and the passenger flow rate is same in one period (Assumption 2); different colours of double arrows in Figure 4 imply different flow control periods. Specifically, the number of controlled passengers is the same in one period. The start time of recording arrival time of passengers at different stations is $7: 02,7: 15,7: 24,7: 35$, and 7:49, respectively, which is shown with green arrows in Figure 4 . The passenger arrival rate on each station at each period $\left(\mathrm{AR}_{s}(t)\right)$ is shown in Table 5. The OD probability matrix for passengers (the ratio of passengers from a station to another $P_{o, s}$ ) is shown in Table 6. Train capacity is 600 , and the number of passengers on platform at the beginning of the study horizon and the capacity on platform is shown in Table 7 .

\subsection{Nonequilibrium Solution by Model I}

5.2.1. Results. To demonstrate the effectiveness of the ISA algorithm, the ISA algorithm and the CPLEX solver are used, respectively, to solve the Model I with same delay penalty functions $\left(h_{1}(\cdot)=\alpha_{1}=0.1, h_{2}(\cdot)=\beta_{1}=0.2\right)$. The Model I will be linearized before solving by CPLEX, and the linearization process is shown in Appendix B. The comparison of the results of the two methods is shown in Table 8 . The optimized result is the solution computed by the ISA algorithm, and the optimal result is the solution that is computed by the CPLEX solver. The ISA algorithm has iterated over 600 times, and the result almost converges when iterating to 500 times (as shown in Figure 5).

The total delay of optimized result is 726 , which is more than that of optimal result 706.5 . The relative gap is $2.68 \%$, which is less than $5 \%$. Besides, the computation time of ISA algorithm is 37.5 seconds, which is only 7 seconds more than that of CPLEX solver, because the scale of numerical study variables is small, which is easily solved by CPLEX solver. But, the range of search in each temperature of ISA algorithm may be small, which may lead to slower convergence and longer computation time. The detailed passenger flow control strategies of optimized and optimal solutions are compared and shown in Table 9. Only a small part of the result data is different, and the difference is not more than 5 . Therefore, the ISA algorithm has high precision.

To demonstrate the effectiveness of the model, the optimized result is compared with the original result without flow control. The Model I is solved by ISA algorithm with the delay penalty functions, $h_{1}(\cdot)=\alpha_{1}=0.1$ and $h_{2}(\cdot)=\beta_{1}=0.2$. The comparison of results is shown in Table 10 .

In the original result, passengers will not be controlled at the entrance, the flow control strategy is not adopted, and the delay of flow control is 0 . Since many passengers were left behind at the platform because of train capacity limitation, it may lead to safety issues. However, in the optimized result, there is no passenger left behind at the platform because of the flow control strategy, though the passengers flow control delay at the entrance still exists. Specifically, compared with the original result, the total delay is reduced by 657 minutes 


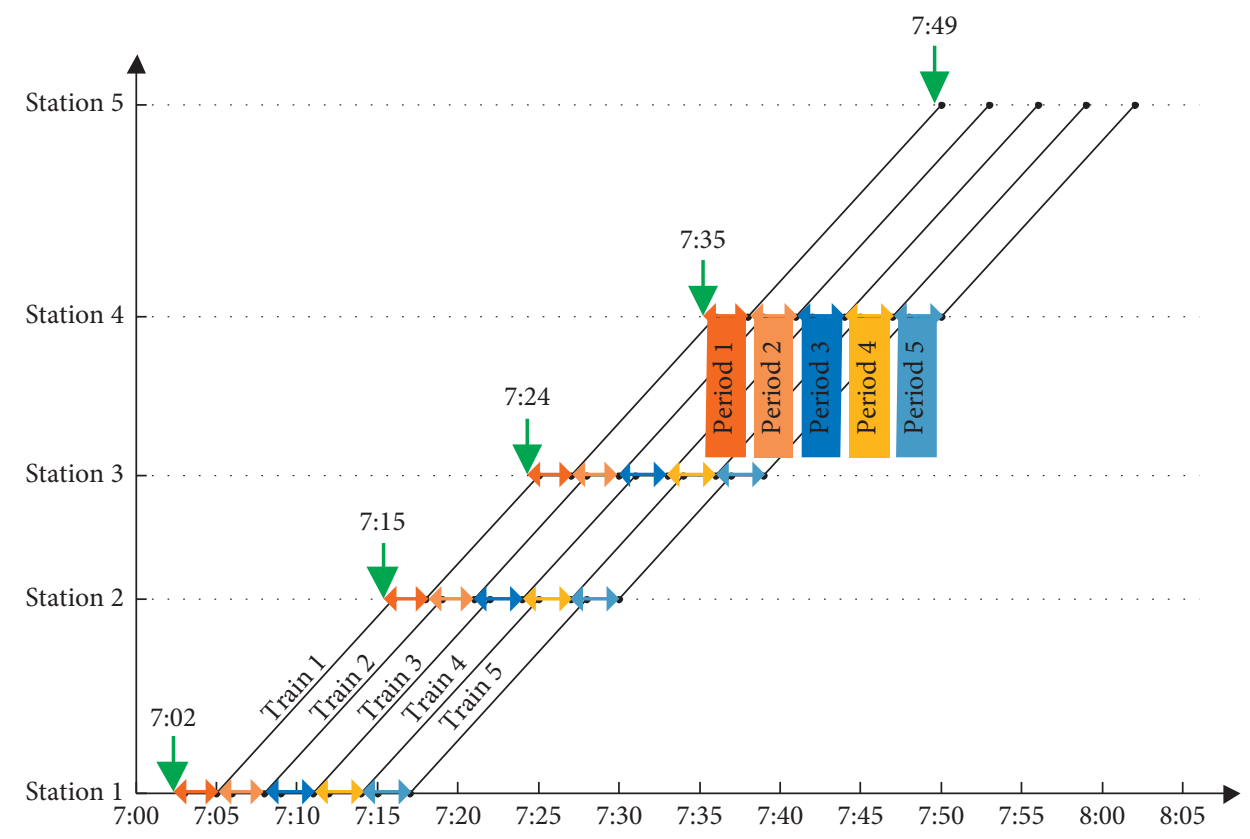

FIgURE 4: The timetable and flow control periods in the numerical study.

TABle 5: The passenger arrival rate (unit: pax/minute).

\begin{tabular}{lccccc}
\hline Flow control period & \multicolumn{3}{c}{ Station } & & \\
& 1 & 2 & 3 & 58 & 90 \\
1 & 72 & 68 & 74 & 89 & 0 \\
2 & 63 & 79 & 83 & 73 & 74 \\
3 & 51 & 62 & 78 & 0 \\
4 & 46 & 50 & 53 & 0 \\
5
\end{tabular}

TABLE 6: The OD probability matrix for passengers (unit: \%).

\begin{tabular}{|c|c|c|c|c|c|}
\hline \multirow{2}{*}{ Station $\mathrm{O}$} & \multicolumn{5}{|c|}{ Station D } \\
\hline & 1 & 2 & 3 & 4 & 5 \\
\hline 1 & - & 20 & 40 & 20 & 20 \\
\hline 2 & - & - & 20 & 40 & 40 \\
\hline 3 & - & - & - & 50 & 50 \\
\hline 4 & - & - & - & - & 100 \\
\hline 5 & - & - & - & - & - \\
\hline
\end{tabular}

TABLE 7: The number of passengers on the platform at beginning and the platform capacity (unit: pax).

\begin{tabular}{|c|c|c|c|c|c|}
\hline \multirow{2}{*}{ Parameters } & \multicolumn{5}{|c|}{ Station } \\
\hline & 1 & 2 & 3 & 4 & 5 \\
\hline Passengers on platform & 100 & 100 & 150 & 150 & 0 \\
\hline Capacity of platform & 1500 & 1500 & 1500 & 1500 & 1500 \\
\hline
\end{tabular}

TABLE 8: Comparison of results for two kinds of methods (unit: minute).

\begin{tabular}{lccccccc}
\hline Result & Methods & $\mathrm{TD}_{\text {control }}$ & $\mathrm{TD}_{\text {left-behind }}$ & $\mathrm{TD}_{\text {total }}$ & $\mathrm{TW}_{\text {platform }}$ & $\mathrm{TW}_{\text {total }}$ & $\mathrm{CT}$ \\
\hline Optimized & ISA & 726 & 0 & 726 & $6,928.5$ & $7,654.5$ & 0.625 \\
Optimal & CPLEX & 706.5 & 0 & 706.5 & $6,928.5$ & 7,635 & 0.508 \\
\hline
\end{tabular}

Notes: CT denotes the computation time. 


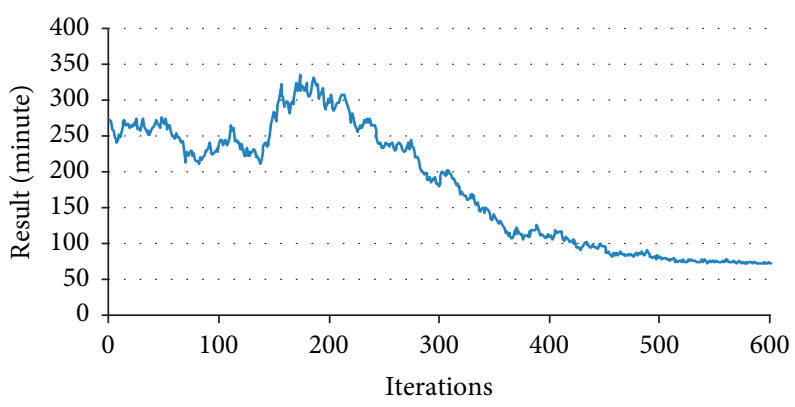

Figure 5: The value of result in each iteration.

TABle 9: The detailed comparison results for two kinds of methods (unit: pax).

\begin{tabular}{|c|c|c|c|c|c|c|}
\hline \multirow{2}{*}{ Flow control period } & \multirow{2}{*}{ Result } & \multicolumn{5}{|c|}{ Station } \\
\hline & & 1 & 2 & 3 & 4 & 5 \\
\hline \multirow{2}{*}{1} & Optimized result by ISA & 7 & 2 & 179 & 119 & 0 \\
\hline & Optimal result by CPLEX & 7 & 2 & 179 & 119 & 0 \\
\hline \multirow{2}{*}{2} & Optimized result by ISA & 2 & 39 & 38 & 85 & 0 \\
\hline & Optimal result by CPLEX & 2 & 39 & 38 & 85 & 0 \\
\hline \multirow{2}{*}{3} & Optimized result by ISA & 0 & 0 & 1 & 0 & 0 \\
\hline & Optimal result by CPLEX & 0 & 0 & 0 & 0 & 0 \\
\hline \multirow{2}{*}{4} & Optimized result by ISA & 0 & 1 & 0 & 1 & 0 \\
\hline & Optimal result by CPLEX & 0 & 0 & 0 & 0 & 0 \\
\hline \multirow{2}{*}{5} & Optimized result by ISA & 5 & 3 & 2 & 0 & 0 \\
\hline & Optimal result by CPLEX & 0 & 0 & 0 & 0 & 0 \\
\hline
\end{tabular}

TABLE 10: The comparison of the optimized and original result (unit: minute).

\begin{tabular}{lccccccc}
\hline Result & $h_{1}(\cdot)$ & $h_{2}(\cdot)$ & $\mathrm{TD}_{\text {control }}$ & $\mathrm{TD}_{\text {left-behind }}$ & $\mathrm{TD}_{\text {total }}$ & $\mathrm{TW}_{\text {platform }}$ & $\mathrm{TW}_{\text {total }}$ \\
\hline Original & - & 1 & 0 & 1,383 & 1,383 & $6,928.5$ \\
Optimized & 0.1 & 0.2 & 726 & 0 & 726 & $6,928.5$ & $7,654.5$ \\
\hline
\end{tabular}

(47.51\%). The flow control strategy is nonequilibrium, since the number of controlled passengers in the first period on stations 3 and 4 are too large, while it is small in other periods at other stations. The strategy fluctuates wildly in terms of both successive periods and successive stations, so it is difficult to implement in practice and may cause continued shut down at some stations. The waiting time at the platform is the same, which means the number of boarding passengers is the same. In summary, although the model provides a flow control strategy which can reduce the number of left-behind passengers at the platform and also reduce the total delay of passengers, it is nonequilibrium.

5.2.2. Sensitivity Analysis of the Model I Parameters. To analyze the sensitivity of the parameters in Model I, some different values of parameters are tested, in which the value of $\alpha_{1}$ is 0.1 and the range of $\beta_{1}$ is $[0.1,0.3]$; then, the value of $\beta_{1}$ is 0.1 and the range of $\alpha_{1}$ is $[0.1,0.3]$. The total delay, flow control delay, and left-behind delay with different parameters are shown in Figure 6. In the test, the left-behind delay is 0 when $1.5 \beta_{1} \geq \alpha_{1}$, in which no passengers are left behind at the station. Besides, the control delay is 0 when $\alpha_{1} \geq 2.5 \beta_{1}$, in which no passengers are controlled at the entrance. When

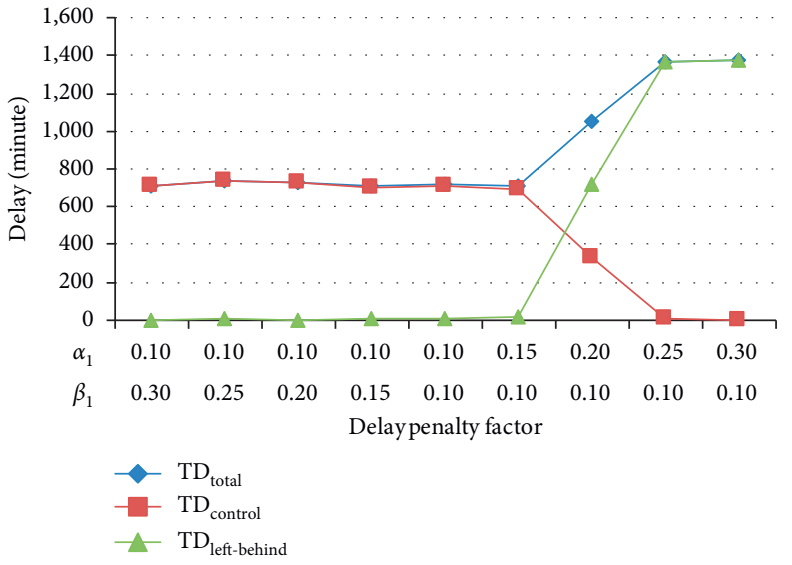

Figure 6: The results of Model I with different parameters.

$\alpha_{1}=0.2$ and $\beta_{1}=0.1$, the flow control delay and left-behind delay are balanced well. Compared with the total delay of these tests, the total delay is minimized when $\alpha_{1} \leq 1.5 \beta_{1}$. Therefore, it is better to control the passengers at the entrance instead of staying at the platform in the case of oversaturation. In summary, the total delay is minimum 
when $\alpha_{1} \leq 1.5 \beta_{1}$, which are the best parameters relationship in the Model I.

\subsection{Equilibrium Solution by Model II}

5.3.1. Results. To avoid the influence of the parameters, we set $\alpha_{1}=\alpha_{2}=0.1$ and $\beta_{1}=\beta_{2}=0.2$ in constant and linear delay penalty functions of Models I and II, respectively. The comparison of results of two models is shown in Table 11.

Compared with the result in Model I, the flow control delay is smaller and the left-behind delay is larger in Model II, and the passenger total delay in constant function test is also smaller than that in Model II. Because more passengers are left behind at the platform instead controlled at the entrance in Model II, the passenger waiting time at the platform in two models is same, which means the number of serviced passengers is same in two strategies. There are added 109.5 minutes in total passenger waiting time in Model II, compared with that in Model I. For the equilibrium indicators; no matter in TEI or in SEI, the value of Model II is smaller than that of Model I, so the result of Model II is more equilibrium than the result of Model I. In summary, the total delay in Model I is smaller than that in Model II, but the flow control delay is reversed, and the strategy from Model II is more equilibrium than that from Model I no matter if it is on temporal or spatial.

The details of passenger distribution at stations in two tests are compared, and the number of controlled passengers, the number of waiting passengers at the entrance and the platform, and the number of left-behind passengers are shown from Figures 7-10, respectively.

Compared with the passenger distribution in the solution of Model I, the controlled passengers at the front stations (such as stations 1 and 2) are more and that at the behind stations (such as stations 3 and 4) are less in the solution of Model II in period 1. In other words, the passengers at the front stations are controlled, and more passengers at the behind stations can boarding the train, because the train capacity will be reserved for the behind stations. This strategy avoids the situation that some stations are controlling too many passengers while other stations do not take any flow control strategy, which can relieve the high-impact flow control strategy at behind stations. Compared with the waiting passengers at the entrance and platform in Model I, the number of waiting passengers at the entrance and platform in Model II is similar between different stations. However, some passengers are left behind at the platform in Model II, due to the weight of left-behind delay is not high enough, which may not be the best parameter relationship in Model II. To analyze the weight of linear functions, sensitivity of parameters in Model II will be analyzed in Section 5.3.2.

In summary, considering the equilibrium of waiting passengers at each station and the passenger patience while waiting at the entrance, it $s$ observed that the solution in Model II is better.

\subsubsection{Sensitivity Analysis of the Model II Parameters.} There are different values of parameters in Model II, which will be tested to analyze the sensitivity of the linear delay penalty functions. In the tests, firstly, the value of $\alpha_{2}$ is set to 0.1 and the range of $\beta_{2}$ is $[0.1,6.0]$; then, the value of $\beta_{2}$ is 0.1 and the range of $\alpha_{2}$ is $[0.1,6.0]$. The total delay, flow control delay, and left-behind delay with different results are shown in Figure 11. The left-behind delay is almost 0 when $\beta_{2} \geq 20 \alpha_{2}$, in which almost no passengers are left behind at the station. Besides, the flow control delay is quite small when $\alpha_{2} \geq 20 \beta_{2}$, in which few passengers are controlled at the entrance. When $1 / 20 \beta_{2} \leq \alpha_{2} \leq 20 \beta_{2}$, the flow control delay and left-behind delay exist. They are balanced while $\alpha_{2}=0.5$ and $\beta_{2}=0.1$. Compared with the total delay of these tests, the total delay is minimized when $\beta_{2} \geq 20 \alpha_{2}$. So, when passengers are oversaturated in the system, they should better be controlled at the entrance instead of left-behind at the platform in Model II. In summary, the total delay is minimum when $\beta_{2} \geq 20 \alpha_{2}$, which are the best parameter relationships in the Model II.

\section{Case Study}

6.1. Introduction of the Case. Beijing Subway Batong Line is one of the lines that operated by the Beijing Metro Company, which connects the central city and Tongzhou suburban area in the east. The layout of Batong Line is shown in Figure 12. It is in east-west direction and has a total length of $18.964 \mathrm{~km}$. The maximum speed is $70 \mathrm{~km} / \mathrm{h}$. It adopts a fixed six-car group with 1,428 service capacity. The down direction is from TUQIAO station to SIHUI station with an operating time of 5:20-23:10. There are 13 stations on the line, of which SIHUI station and SIHUI East station are interchange stations with Line 1.

As an important commuting line for the passengers of the Tongzhou District, the "tidal phenomenon" of passenger flow is obvious in the Batong line. More than $98 \%$ of passengers are in the down direction (TUQIAO Station-SIHUI Station) in the morning peak period, and most of the passengers are commuters. Batong Line is also one of the busiest lines in the network, both the station and the carriage are seriously crowded, and there are more than 40,000 pax/ hour in the down direction in the morning peak period. As a result, 8 stations of Batong line were controlled to carry out flow control strategy in morning peak period since 2015, so we select the down direction of the Batong Line and use the 7:00 9:00 (120 minutes horizon) of the morning peak on May 20, 2015 (Wednesday) as a case. Except for the terminal station (Sihui station), the platforms of all stations on the Batong line are side type instead of island type, which is easy to control the inflow in down direction by setting up the fencing facility at the halls. The arrival time of train 1 at each station of Batong Line is shown in Table 12. The train departure headway $f$ is 3 minutes; then we can get the train departure time parameters $L_{s, r}(t)$. The capacity of platform at each station $\operatorname{Cap}_{s}^{P}$ is shown in Table 12, which is the product of the platform active area and the maximum passenger gathering density. The platform active area is the passenger usable area that is the total platform area minus the occupied area by the infrastructures, and the data are from the on-site survey. The maximum passenger gathering density is $2 \mathrm{pax} / \mathrm{m}^{2}$, because the passengers may be in 
TABLE 11: Comparison of result for two forms of penalty functions (unit: minute).

\begin{tabular}{lccccrrr}
\hline Model & TD $_{\text {control }}$ & TD $_{\text {left-behind }}$ & TD $_{\text {total }}$ & TW $_{\text {platform }}$ & TW $_{\text {total }}$ & SEI & TEI \\
\hline Model I $(0.1,0.2)$ & 726 & 0 & 726 & $6,928.5$ & $7,654.5$ & 21.50 & 17.04 \\
Model II $(0.1,0.2)$ & 724.5 & 111 & 835.5 & $6,928.5$ & 7,764 & 9.07 & 10.67 \\
\hline
\end{tabular}

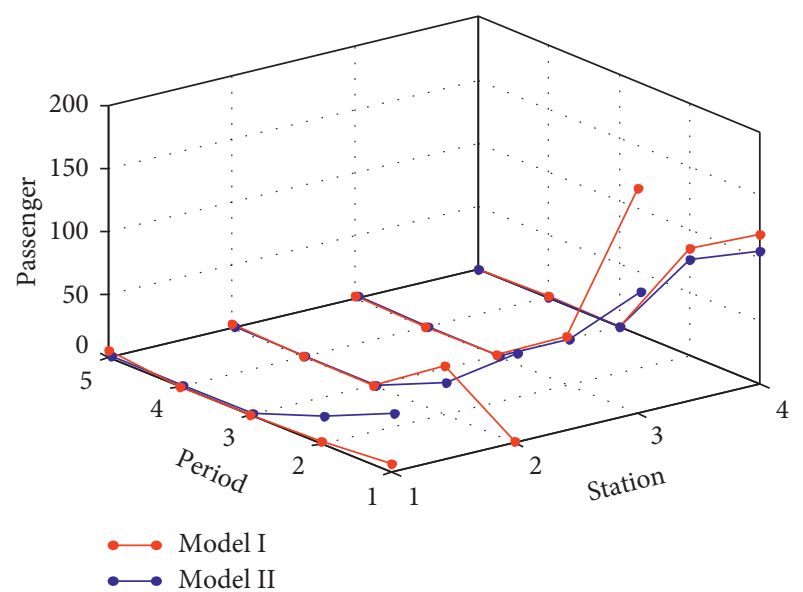

FIgURE 7: Comparison of controlled passengers in two models.

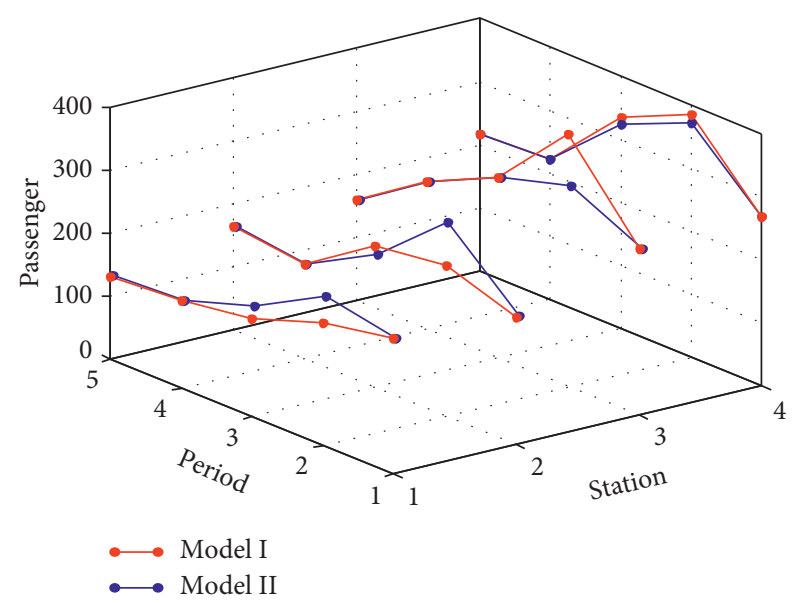

FIGURE 8: Comparison of waiting passengers at the entrance in two models.

various kinds of contact, extremely uncomfortable, and even have a collective panic phenomenon when the density is larger than that [29].

Passenger demand is collected by the Automatic Fare Collection (AFC) system, which includes the swiping data of passengers. However, the number of entering passengers in peak hours may be reduced because the swiping data are under the original follow control strategy that the operator controlled with their managerial experience. To recover the real passenger arrival rate at the entrance, the swiping data were magnified 1.2 times; then, we can get the passenger arrival rate at each station in each time $\left(\mathrm{AR}_{s}(t)\right)$. To show the data conveniently, it is handled by the number of

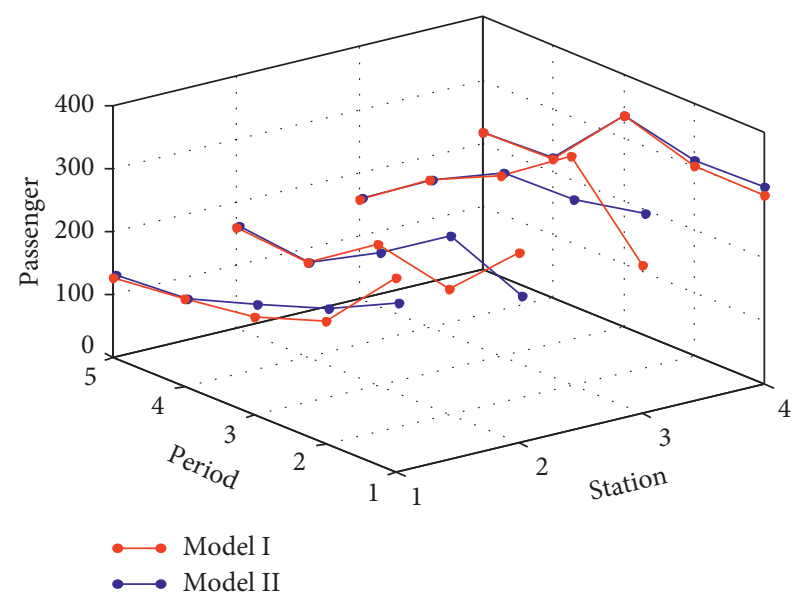

FIGURE 9: Comparison of waiting passengers at the platform in two models.

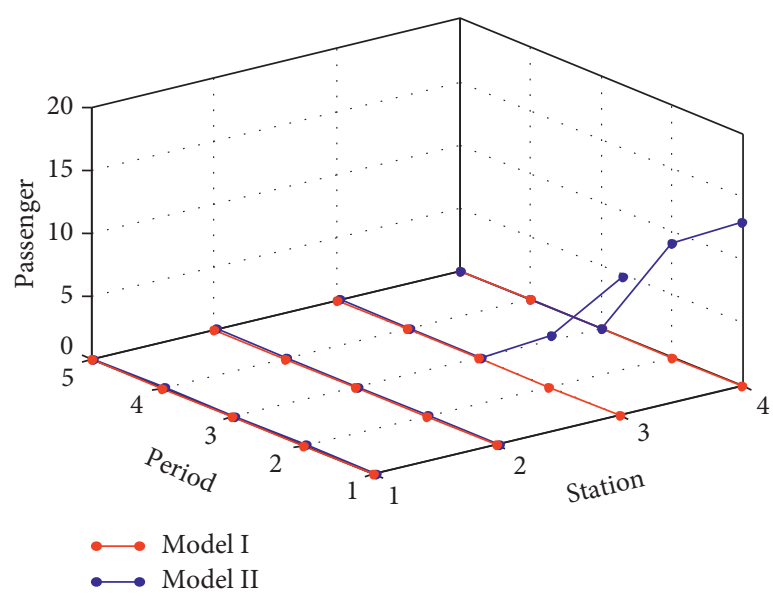

FIGURE 10: Comparison of left-behind passengers at the platform in two models.

entering passengers with 15 minutes granularity at every station and it is shown in Table 13.

The OD probability matrix for passengers (the ratio of passengers from a station to another $P_{o, s}$ ) is shown in Table 14 that there are more than $85 \%$ passengers of each stations (except for SIHUI East station) who exit or transfer on SIHUI East station and SIHUI station. Because there are few of passengers alight at intermediate station and the train capacity is limited, the passengers are difficult to board and many passengers are left behind on the platform at downstream stations. To keep the metro system operation safety, the passenger flow control strategy is necessary to apply. The Batong Line starts to operate at 5:20, so we can calculate the number of passengers at the platform by the AFC data. We 


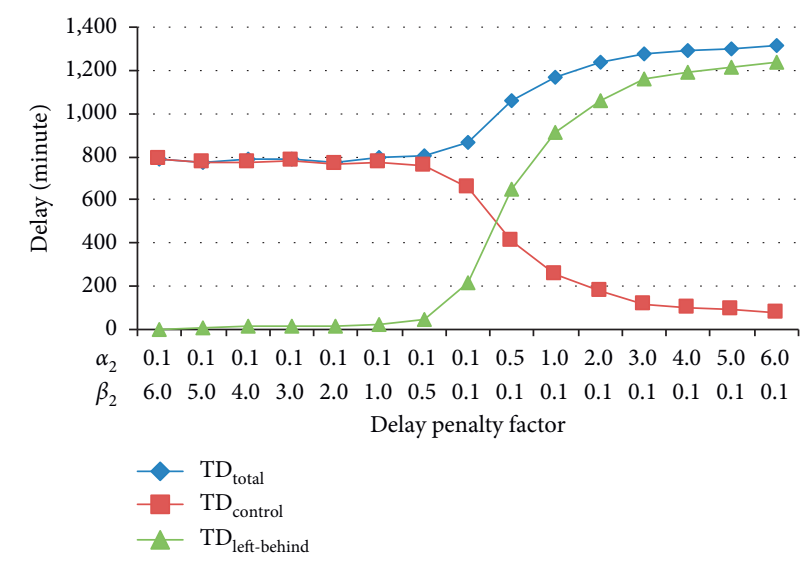

Figure 11: The results of Model II with different parameters.

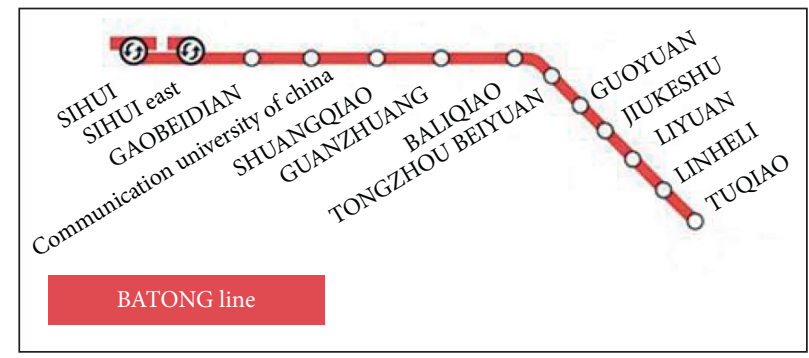

(93) Interchange station

Intermediate station

Figure 12: The layout of Batong line.

TABle 12: The first train's arrival time and the platform capacity at each station.

\begin{tabular}{lccc}
\hline No. & Station & Train 1 arrival time & Platform capacity (pax) \\
\hline 1 & TUQIAO & $7: 00$ & 894 \\
2 & LINHELI & $7: 01$ & 867 \\
3 & LIYUAN & $7: 04$ & 834 \\
4 & JIUKESHU & $7: 06$ & 867 \\
5 & GUOYUAN & $7: 08$ & 857 \\
6 & TONGZHOU BEIYUAN & $7: 11$ & 844 \\
7 & BALIQIAO & $7: 13$ & 936 \\
8 & GUANZHUANG & $7: 17$ & 810 \\
9 & SHUANGQIAO & $7: 20$ & 793 \\
10 & Communication University of China & $7: 23$ & 900 \\
11 & GAOBEIDIAN & $7: 26$ & 806 \\
12 & SIHUI East & $7: 28$ & 1,106 \\
13 & SIHUI & $7: 31$ & 1,029 \\
\hline
\end{tabular}

find that no passengers will be left behind at the platform before 7:00 because of the sufficient train capacity. Therefore, there are no passengers at the platform at the beginning of study time horizon. The other necessary parameters of train and line are shown in Table 15.

6.2. Results. To demonstrate the effectiveness of the Model I and Model II and the ISA algorithm, four experiments are designed with different conditions and parameters, which are shown in Table 16. Experiment A is the original solution without any flow control strategy. The Model II with best parameters relationship is used in Experiment B, from which can get the best equilibrium result. The Model I with best parameters relationship is also used in Experiment C, which can produce the best nonequilibrium result. The Model II with the same parameters of Experiment $C$ is tested in Experiment $\mathrm{D}$ to compare with the nonequilibrium result. The results of four experiments are listed in Table 17. 
TABle 13: Passenger demand of Batong line (7:00-9:00) (unit: pax/minute).

\begin{tabular}{|c|c|c|c|c|c|c|c|c|c|c|c|c|}
\hline \multirow{2}{*}{ Period } & \multicolumn{12}{|c|}{ Station } \\
\hline & 1 & 2 & 3 & 4 & 5 & 6 & 7 & 8 & 9 & 10 & 11 & 12 \\
\hline $7: 00-7: 15$ & 101 & 29 & 60 & 25 & 45 & 35 & 13 & 46 & 59 & 43 & 6 & 41 \\
\hline $7: 15-7: 30$ & 108 & 36 & 74 & 34 & 76 & 54 & 25 & 55 & 64 & 57 & 8 & 54 \\
\hline $7: 30-7: 45$ & 119 & 50 & 78 & 43 & 73 & 57 & 25 & 75 & 91 & 73 & 8 & 72 \\
\hline $7: 45-8: 00$ & 113 & 51 & 87 & 45 & 77 & 49 & 29 & 76 & 79 & 76 & 12 & 90 \\
\hline $8: 00-8: 15$ & 86 & 41 & 102 & 45 & 84 & 74 & 25 & 69 & 84 & 64 & 11 & 81 \\
\hline $8: 15-8: 30$ & 75 & 30 & 65 & 33 & 62 & 50 & 20 & 67 & 62 & 60 & 11 & 84 \\
\hline $8: 30-8: 45$ & 61 & 25 & 66 & 30 & 54 & 49 & 17 & 44 & 52 & 46 & 8 & 73 \\
\hline 8:45-9:00 & 46 & 19 & 49 & 23 & 53 & 45 & 16 & 44 & 54 & 45 & 9 & 55 \\
\hline
\end{tabular}

TABLE 14: The OD probability matrix for passengers on Batong line (unit: \%).

\begin{tabular}{|c|c|c|c|c|c|c|c|c|c|c|c|c|c|}
\hline \multirow{2}{*}{$\mathrm{O}$} & \multicolumn{13}{|c|}{$\mathrm{D}$} \\
\hline & 1 & 2 & 3 & 4 & 5 & 6 & 7 & 8 & 9 & 10 & 11 & 12 & 13 \\
\hline 1 & - & 0.1 & 0.1 & 0.4 & 0.3 & 1.2 & 0.3 & 1.6 & 2.5 & 0.8 & 3.4 & 63.0 & 26.2 \\
\hline 2 & & - & 0.2 & 0.8 & 0.3 & 1.2 & 0.2 & 1.5 & 2.4 & 0.8 & 3.2 & 63.2 & 26.3 \\
\hline 3 & & & - & 0.3 & 0.2 & 0.7 & 0.2 & 1.1 & 1.9 & 0.9 & 3.2 & 65.8 & 25.6 \\
\hline 4 & & & & - & 0.1 & 0.6 & 0.1 & 1.2 & 1.9 & 0.8 & 2.7 & 66.2 & 26.4 \\
\hline 5 & & & & & - & 0.2 & 0.1 & 1.0 & 1.6 & 1.0 & 3.3 & 65.8 & 27.1 \\
\hline 6 & & & & & & - & 0.0 & 0.7 & 3.6 & 1.1 & 4.7 & 65.2 & 24.8 \\
\hline 7 & & & & & & & - & 0.3 & 0.8 & 0.8 & 2.0 & 65.9 & 30.3 \\
\hline 8 & & & & & & & & - & 0.3 & 0.2 & 2.0 & 68.3 & 29.2 \\
\hline 9 & & & & & & & & & - & 0.1 & 0.4 & 71.7 & 27.8 \\
\hline 10 & & & & & & & & & & - & 0.2 & 72.5 & 27.3 \\
\hline 11 & & & & & & & & & & & - & 66.7 & 33.3 \\
\hline 12 & & & & & & & & & & & & - & 100 \\
\hline 13 & & & & & & & & & & & & & - \\
\hline
\end{tabular}

TABLE 15: Necessary parameters.

\begin{tabular}{lcc}
\hline Parameters & Meaning & Value \\
\hline$T_{\text {total }}$ & Total passenger flow control time horizon & 120 minutes \\
Temp $S$ & Start temperature & $90^{\circ} \mathrm{C}$ \\
$\Delta$ Temp & Cooling rate & 0.99 \\
Temp $E$ & End temperature & $1^{\circ} \mathrm{C}$ \\
$\rho$ & Precision of disturbance & 0.01 \\
$\Delta t$ & Duration of each flow control period & 3 minutes \\
\hline
\end{tabular}

TABLE 16: Four experiments.

\begin{tabular}{lcccc}
\hline Experiment & With flow control strategy or not & Model & Parameters & Solution \\
\hline Experiment A & No & - & - & Solution A \\
Experiment B & Yes & Model II & $\alpha_{2}=0.1, \beta_{2}=6.0$ & Solution B \\
Experiment C & Yes & Model I & $\alpha_{1}=0.1, \beta_{1}=0.2$ & Solution C \\
Experiment D & Yes & Model II & $\alpha_{2}=0.1, \beta_{2}=0.2$ & Solution D \\
\hline
\end{tabular}

TABLE 17: Comparison of the results (unit: minute).

\begin{tabular}{|c|c|c|c|c|c|c|c|}
\hline Solution & $\mathrm{TD}_{\text {control }}$ & $\mathrm{TD}_{\text {left-behind }}$ & $Z$ & $\mathrm{TD}_{\text {total }}$ & $\mathrm{TW}_{\text {platform }}$ & $\mathrm{TW}_{\text {total }}$ & $\mathrm{CT}$ \\
\hline Solution A & - & 162,324 & - & 162,324 & 91,227 & 253,551 & - \\
\hline Solution B & $81,667.5$ & 75 & $1,167,628$ & $81,742.5$ & 91,227 & $172,969.5$ & 61.6 \\
\hline Solution C & $81,685.5$ & 3 & $8,169.2$ & $81,688.5$ & 91,227 & $172,915.5$ & 56.1 \\
\hline Solution D & $79,738.5$ & 3,375 & $1,193,920.7$ & $83,113.5$ & 91,227 & $174,340.5$ & 67.0 \\
\hline
\end{tabular}

Notes: CT denotes the computation time. 


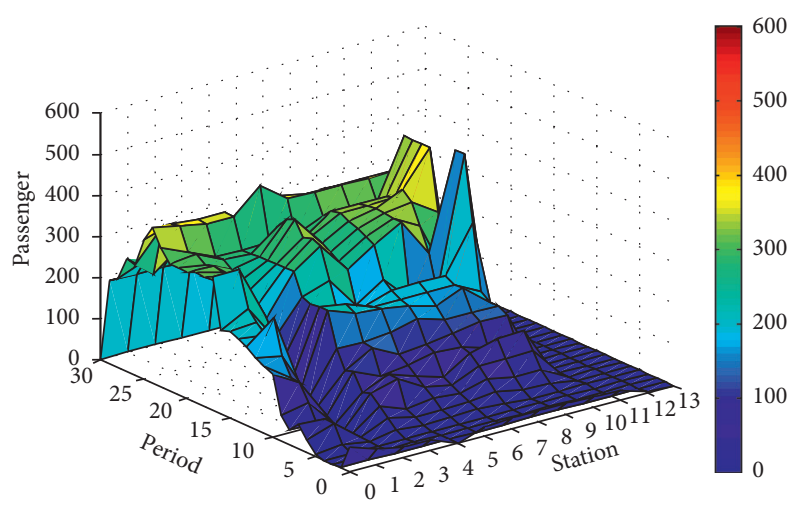

(a)

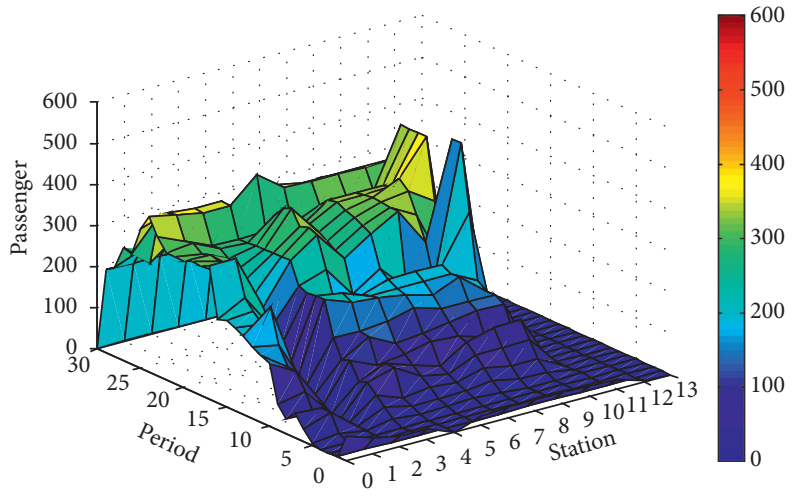

(b)

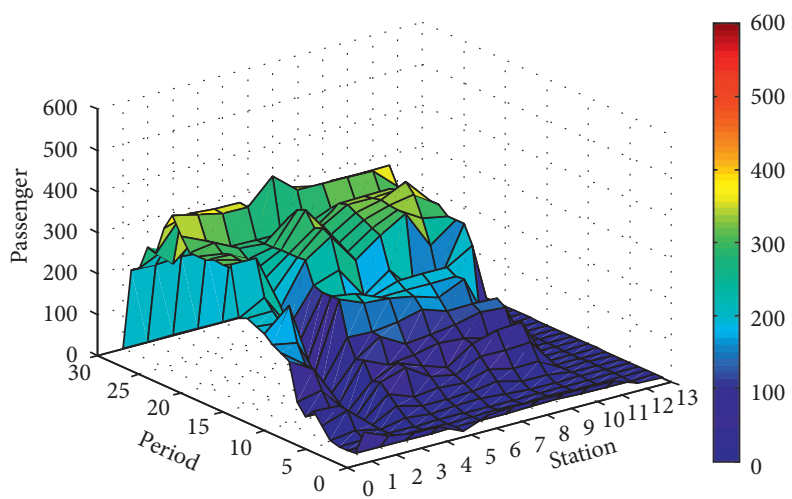

(c)

FIgURE 13: The number of controlled passengers with different models. (a) Solution B. (b) Solution C. (c) Solution D.

In experiment B, the Model II is used to optimal the passenger flow control strategy, in which the linear delay penalty functions are $\alpha_{2}=0.1, \beta_{2}=6.0$. The ISA algorithm is used to solve the model, and it takes 61.6 minutes.

Solution B is obtained by Model II $\left(\alpha_{2}=0.1, \beta_{2}=6.0\right)$, in which the left-behind delay is quite small (75 minutes) and the total delay is almost the flow control delay $(81,667.5$ minutes). The flow control strategy at each station in each period is shown in Figure 13. In experiment A, the original result, that is, the solution $\mathrm{A}$ without any passenger flow control strategy is calculated, in which the total delay is the passengers' left-behind delay (162,324 minutes). Compared with the solution A, the total delay is decreased to 80,581.5 minutes, which is decreased by $49.64 \%$. Besides, the total waiting time of passengers is also saved $80,581.5$ minutes in solution B. In summary, the proposed equilibrium passenger flow control model can provide an effective flow control strategy, in which the passenger delay and waiting time can be greatly decreased.

To analyze the difference of the Model I and Model II, the two models with same parameters $\left(\alpha_{1}=\alpha_{2}=0.1\right.$; $\beta_{1}=\beta_{2}=0.2$ ) are used in the experiments $C$ and $D$, which get solutions $\mathrm{C}$ and $\mathrm{D}$, respectively. Compared with the solution C, the left-behind delay (3,375 minutes) is sacrificed to reduce the flow control delay $(79,738.5$ minutes) in solution $\mathrm{D}$, and the total delay is increased 1,425 minutes. Compared with solution B, more flow control delay is used and less left-behind delay is used in solution C, and total delay is decreased 54 minutes. Furthermore, the computation time is least in solution C.

To testify the efficiency of the ISA algorithm, the Experiment $\mathrm{C}$ with the linearized Model $\mathrm{I}$ is also solved by CPLEX solver. With 5 hours computation, the gap between the feasible solution and the relaxed solution is still larger than $10 \%$, and the objective function of the feasible solution is 8,726.5, which is larger than the result of ISA algorithm (Solution C). Therefore, the ISA algorithm is efficient.

The two equilibrium performance indicators of flow control strategies with solutions $\mathrm{B}, \mathrm{C}$, and D are shown in Table 18, which also include the maximum spatial equilibrium indicator in the different periods $\left(\mathrm{SEI}_{t}\right)$ and the maximum variation of controlled passengers at different stations in different periods $\left(\bar{q}^{\text {control }}(s, t)\right)$.

For SEI, solutions B and D are smaller than solution C, which means the results of Model II are more equilibrium on spatial and the number of controlled passengers is similar in different stations for each period. Considering the maximum $\mathrm{SEI}_{t}$, solutions B and D are smaller, which means the strategy of Model II are still equilibrium on spatial even in the worst period. For TEI, solution B and D is smaller than solution C, which means the results of Model II are more equilibrium on temporal and the fluctuation of passengers in different periods is smooth. Consider the maximum $\bar{q}^{\text {control }}(s, t)$, solutions B and D are smaller, which means the strategy of 
TABLE 18: Equilibrium performance indicators of results.

\begin{tabular}{lcccc}
\hline Solution & SEI & $\max \operatorname{SEI}_{t}$ & TEI & 13.01 \\
\hline Solution B & 78.86 & 155.39 & 45.80 & 107.50 \\
Solution C & 100.91 & 186.94 & 15.53 & 225.50 \\
Solution D & 76.24 & 148.58 & $\bar{q}^{\text {control }}(s, t)$ \\
\hline
\end{tabular}

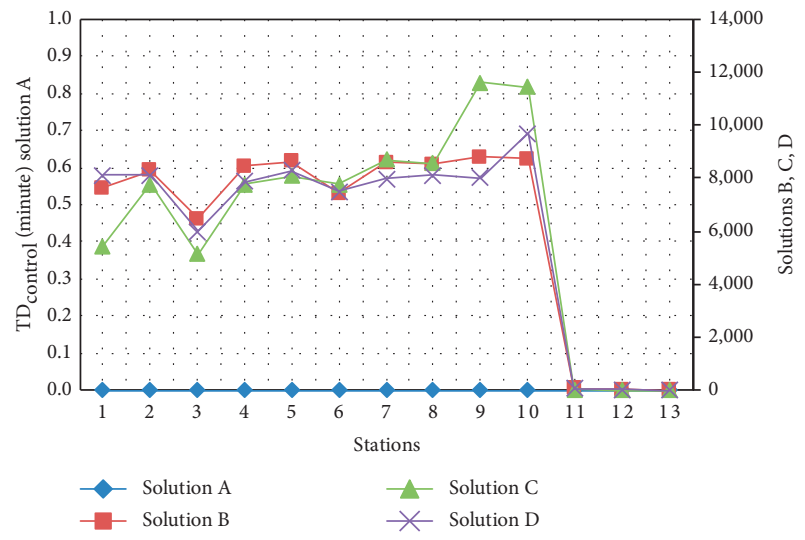

(a)

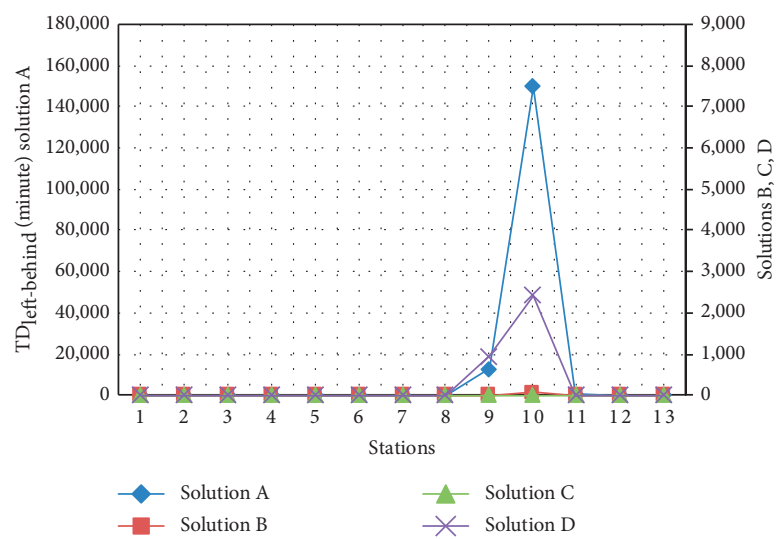

(b)

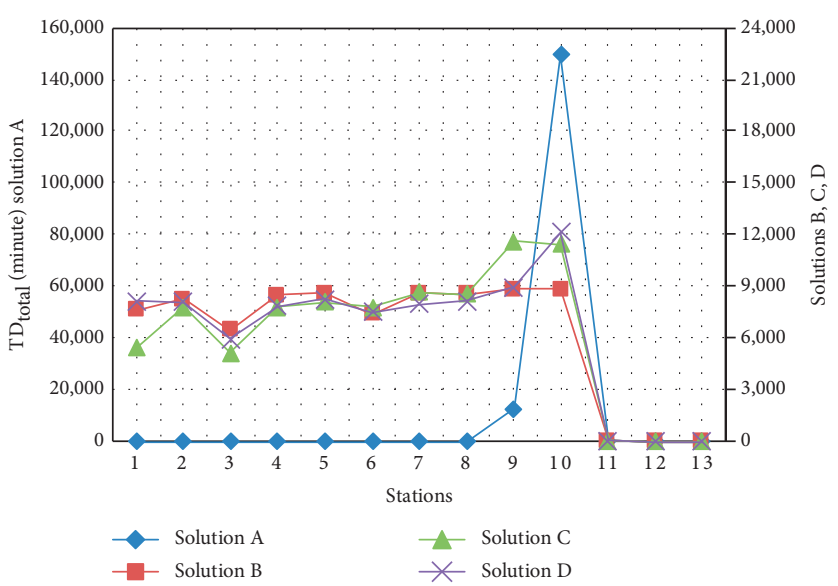

(c)

FIgURe 14: The delay indicators on each station of each solution. (a) Flow control delay. (b) Left-behind delay. (c) Total delay.

Model II is still equilibrium on temporal even in the worst situation. In summary, the strategy of Model II is equilibrium no matter on spatial or temporal.

To analyse the equilibrium of strategy on spatial and temporal in detail, the flow control strategies of solutions $\mathrm{B}$, $\mathrm{C}$, and $\mathrm{D}$ at each station in each period are shown in Figures 13(a)-13(c). In solution $C$, in the same period, the number of controlled passengers at successive stations is quite different, in which some stations control over 500 passengers in a period and some stations just control less than 100 passengers. That makes the passengers at different stations feel unfair. Furthermore, at the same station, the number of controlled passengers at different successive periods fluctuates wildly, which is nonequilibrium, so the flow control strategy of solution $\mathrm{C}$ cannot be used in practice. While, in solutions $\mathrm{B}$ and $\mathrm{D}$, the problems in solution $\mathrm{C}$ can be solved. In the same period, the number of controlled passengers is similar at successive stations (expect for the last two transfer stations because the train have enough capacity at last two stations), which can avoid some stations controlling a lot and most of the stations just controlling no more than 350 passengers in a period. Besides, at the same station, the number of controlled passengers is rising as time goes on, but the change of controlled passengers is relatively smooth, which is an equilibrium result and easy to carry out in reality. Compared with the solution $\mathrm{D}$, the flow control strategy of solution $\mathrm{C}$ is more strictly (left-behind passengers are less), so the controlled passengers are more in each period at each station. In summary, the result of constant delay penalty factor is better than linear one, but the flow control strategy is nonequilibrium, which is difficult to be used in reality. The flow 


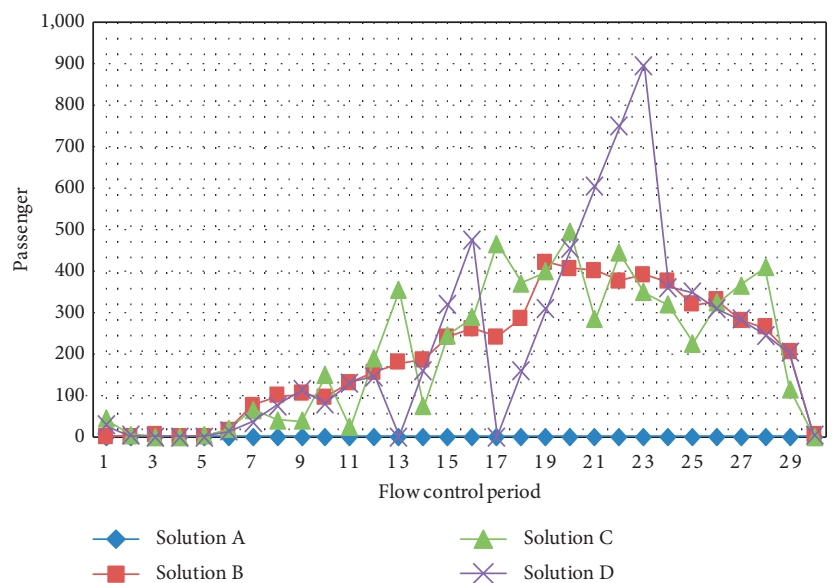

(a)

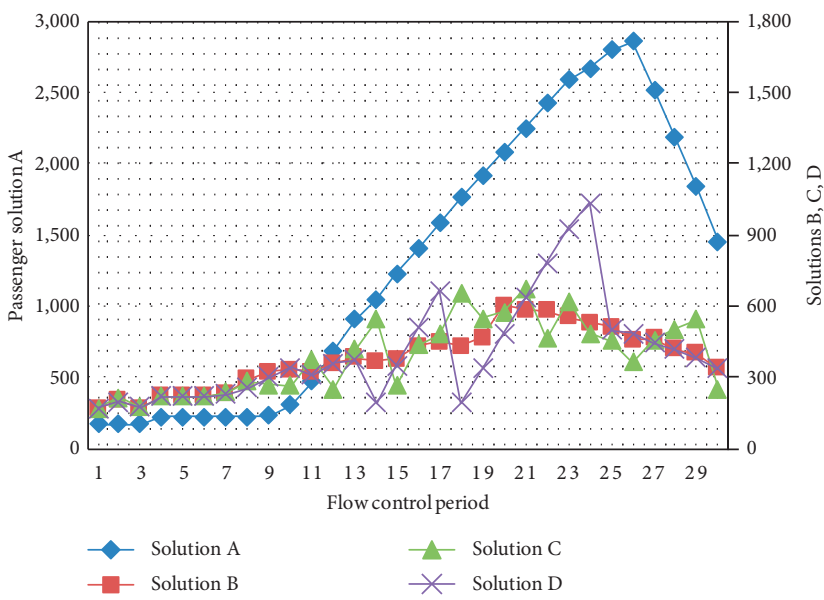

(c)

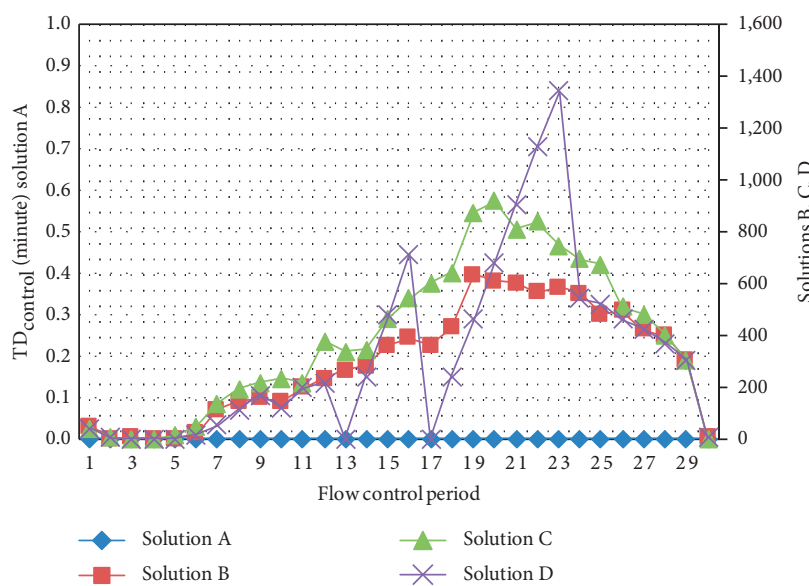

(e)

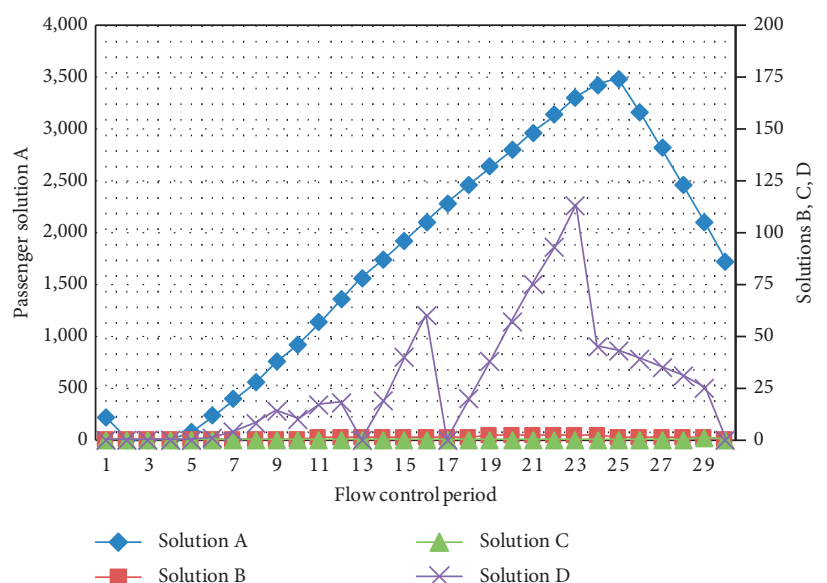

(b)

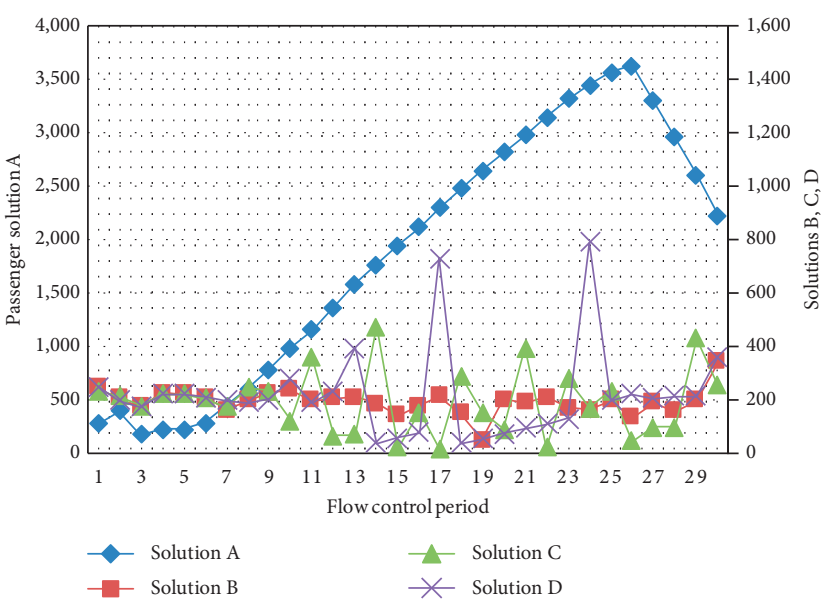

(d)

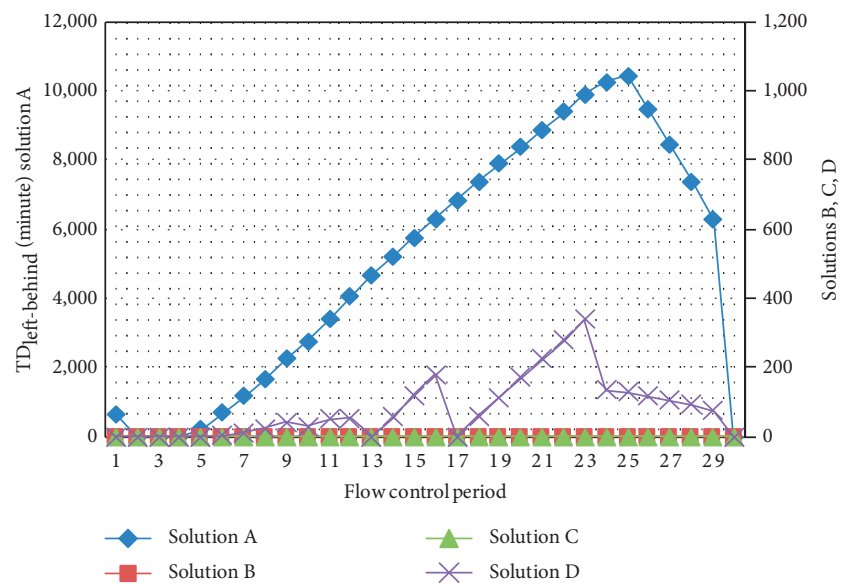

(f)

Figure 15: Continued. 


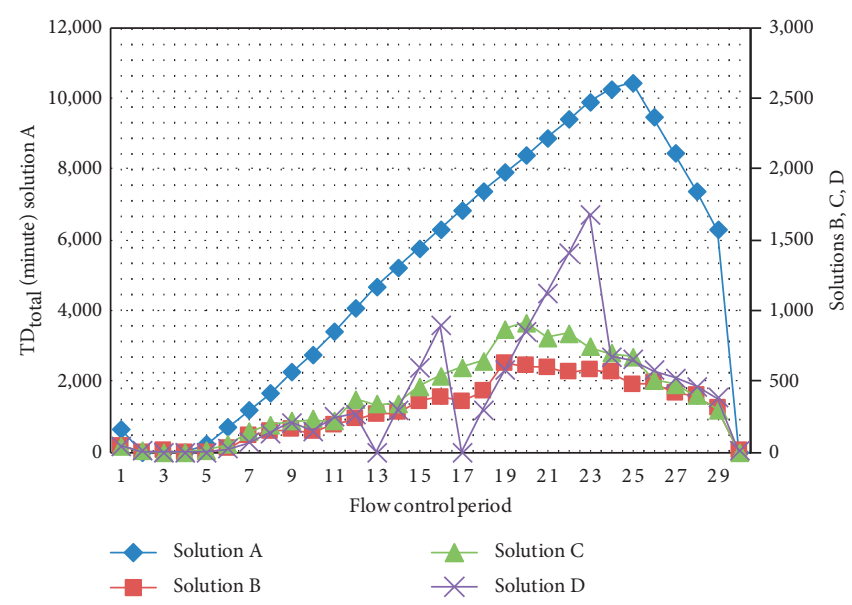

(g)

Figure 15: Seven indicator comparison with different solutions on Communication University Station in each period. (a) Controlled passengers at entrance. (b) Left-behind passengers at the platform. (c) Waiting passengers at entrance. (d) Waiting passengers at platform. (e) Flow control delay of passengers at the entrance. (f) Left-behind delay of passengers at the platform. (g) Total delay of passenger travel time.

control strategy with linear delay penalty function is equilibrium. It not only balances the number of controlled passengers at different stations but also is easy to be used in reality.

As shown in Figure 14, the flow control delay, left-behind delay, and total delay of each station are compared to analysis the delay time on each station of each solution. Compared with solution A, the total delay and the leftbehind delay of station 10 are decreased and the flow control delay of previous stations (stations 1-9) is increased in solutions B, C, and D. Specifically, compared with the result of solution A (without any flow control strategy), the total delay on station 10 is greatly alleviated in the solutions $\mathrm{B}, \mathrm{C}$, and $\mathrm{D}$ (with the flow control strategy from the proposed models), which is borne by the previous stations equally. Furthermore, compared with the result of solution $\mathrm{C}$ by the Model I, the total delay is more equally from station 1 to station 10 in solution B by the Model II, in which the station total delay is closer to the average total delay at stations 1, 9, and 10 in solution $\mathrm{B}$. Therefore, the proposed equilibrium flow control model (Model II) can control the delay of different stations more equal, so that some situations which may lead to extensively long delays for a small number of stations (stations 9 and 10) were avoided.

6.3. Analysis of Different Solutions at a Station. In reality, the Communication University of China station is one of the most stations with left-behind passengers, not only because of the large passenger demand at this station but also because the train loading rate is almost saturated before arriving at this station. Therefore, seven critical indicators will be discussed, which includes four number of passengers indicators (the number of controlled passengers at the entrance, the number of left-behind passengers at the platform, and the number of waiting passengers at entrance and platform in each period at this station) and three delay

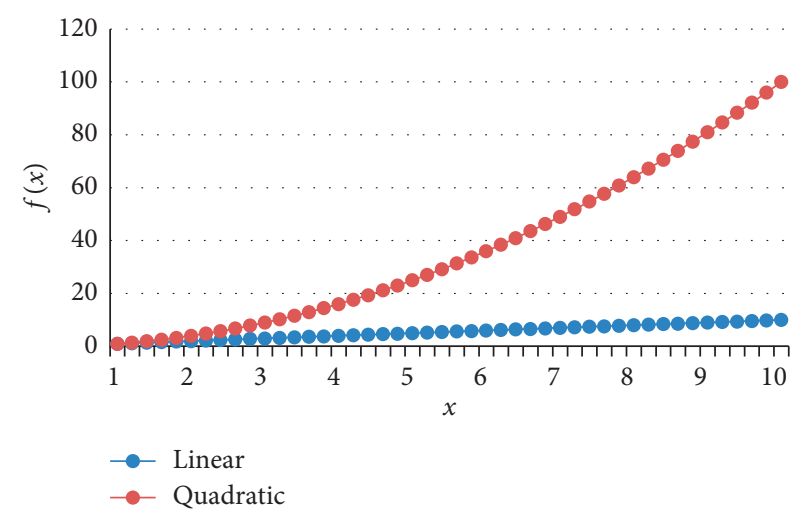

Figure 16: The comparison of linear and quadratic functions.

indicators (the flow control delay at the entrance, the leftbehind delay at the platform, and the total delay of passenger travel time in each period at this station). The seven indicators of solutions A, B, C, and D are shown in Figures 15(a)-15(g), respectively.

In Figure 15(a), the number of controlled passengers in solutions C and D fluctuates wildly, which is a nonequilibrium flow control strategy. In solution $B$, the controlled passengers are rising and then falling down smoothly, which is an equilibrium strategy for real-word.

Consider the left-behind passengers at the platform (in Figure 15(b)), almost no passengers are left behind in solutions $\mathrm{B}$ and $\mathrm{C}$, while there are more than 500 passengers are left behind in solution $\mathrm{D}$ in study time horizon. Therefore, the flow control strategies of solutions B and C can effectively avoid the situation that passengers are left behind at the platform.

At the entrance (Figure 15(c)), in solution D, over 700 passengers are waiting at the entrance in period 22-24, which may lead to the entrance overcrowding. But, in solutions $\mathrm{B}$ and $\mathrm{C}$, the number of waiting passengers at the entrance is 
from 200 to 600 , which is relatively moderate. Therefore, the solutions $\mathrm{B}$ and $\mathrm{C}$ can avoid the crowded queue at the entrance.

At the platform (Figure 15(d)), over 700 passengers are waiting in periods 17 and 24 in solution D and over 300 passengers are waiting in some periods in solution $\mathrm{C}$, which may lead to the platform overcrowding. While, in solution $B$, no more than 250 passengers are waiting at the platform in each period. Therefore, the solution B can avoid overcrowding at the platform.

Then, as shown in Figures 15(e) $-15(\mathrm{~g})$, three kinds of delay in different solutions are compared. As time goes on, the left-behind delay and the total delay of solution $\mathrm{A}$ increase rapidly and over 10,000 minutes in periods 24 and 25, which is far greater than the value in other solutions (the delay is not exceeding 2,000 minutes). Therefore, compared with the solution without any flow control strategy (solution A), the solution from the proposed models (solutions B, C, and $\mathrm{D}$ ) can reduce the passenger left-behind delay and the total delay greatly at specific stations. The situation that may lead to extensively long delays for a station can be avoided. Furthermore, compared with the flow control delay or the total delay in solution $\mathrm{C}$, the results in solution $\mathrm{B}$ is smaller in periods 5-28. It means that the flow control strategy from the Model II (with the best parameters relationship) can reduce the passenger left-behind delay and the total delay at a specific station better than that from the Model I (with the best parameters relationship).

In summary, the equilibrium passenger flow control strategy of solution B can not only avoid the overcrowding situation at the entrance and the platform but also be easily implemented in practice.

\section{Conclusion}

Passenger flow control is one of the most effective methods to alleviate passenger crowd in station for an oversaturated urban rail transit system, especially when the train frequencies cannot be further increased due to the signal system. In this study, an optimum passenger flow control model was constructed for a line, in which the line is unidirectional or the line is bidirectional, but the inflow with opposite directions can be controlled, respectively. The objective function was to minimize the total passenger delay, which consists of the delay of passenger flow control at the entrance and the delay of the passenger left-behind at the platform. The academic contribution of the model is that flow control strategies are controlled by delay penalty functions. By applying different forms of delay penalty functions, i.e., constant and linear, nonequilibrium and equilibrium solutions can be obtained. Based on the characteristics of the model, the ISA algorithm was proposed, which was based on the random disturbance operator and could solve the model with high accuracy and high efficiency.

A numerical study was introduced to validate the model and the algorithm; the results by CPLEX and the ISA algorithm were compared, which illustrated the accuracy of the ISA algorithm. Compared with the original result without any flow control strategy, the total delay decreased 657 minutes $(47.51 \%)$ in optimized result. Therefore, the flow control strategy can decrease the total delay. Compared with the nonequilibrium solution by Model I, the equilibrium solution by Model II can balance the number of controlled passengers at successive stations and in successive periods. Meanwhile, the sensitivities of the parameters in Model I and Model II were analyzed, and the best parameters relationship could be confirmed: the total delay was minimum when $\alpha_{1} \leq 1.5 \beta_{1}$ in Model I and $\beta_{2} \geq 20 \alpha_{2}$ in Model II.

A real-world case from Batong Line railway corridor in Beijing urban rail transit system was used to further compare the strategies of two models and test the applicability of the algorithm in practice. The best parameter relationship in Model I and Model II and the two models with same parameters were tested and discussed. The result showed that the result in Model I was better than Model II no matter in total delay time or in total waiting time, but the flow control strategy of Model I was nonequilibrium, which is difficult to be used in reality. The flow control strategy of Model II was equilibrium, which can balance well between successive stations and successive periods. The flow control strategies with different solutions in Communication University of China station were compared.

The result shows that the optimum equilibrium flow control model not only avoids the overcrowding situation at the entrance and platform but also balances the number of controlled passengers in successive periods. This can significantly improve the flow control strategy and facilitate the implementation. It concludes that the model is effective for practical use.

In the future, the research should be studied in the following aspects: (i) the passenger arrival rate and the proportion of passengers from original station to the destination station should be more accurate. (ii) The equilibrium between stations may result in a situation that some stations with few passengers are attached great attention, which is inconsistent with reality. Therefore, more types of fairness should be discussed, such as proportion fairness. If the number of controlled passengers is proportional to the number of arrived passengers, it is another fairness and may consistent with the reality. (iii) To realize (ii), more types of delay penalty functions should be discussed, such as triple, exponent, power, and logarithm functions. (iv) The passengers from different directions on a line should be considered, and the synchronous passenger flow control of the network should be discussed, in which the path selection behaviour of passengers should also be considered.

\section{Appendix}

\section{A. The significance of Model II}

Before we explain the significance of quadratic objective function in Model II, the difference between linear function and quadratic function in resource allocation problem will be discussed.

Firstly, a simple example about resource allocation problem is proposed. On spatial allocation, the temporal 
allocation is ignored, which means that the analyzed case is in a period of time. There are $I+1$ stations on the line with Cap corridor transit capacity in one direction. We assume that the number of arrived passenger at station $i$ is $x_{i}$, which is no less than Cap/I and their destination is $I+1$ station. To meet the oversaturated demand with limited capacity, the resource should be allocated to each station.

A linear model (LM) is proposed and shown as follows, which has a linear objective function $f_{1}(x)$ with two constraints (equations (A.2) and (A.3)). And, the best result is obvious, $f_{1}(x)=$ Cap, with the solution that any $x_{i}$ meet $\sum_{i \in I} x_{i}=$ Cap. The allocation result is not decided by the objective function. For example, a solution $\left(x_{1}=\right.$ Cap, $\left.x_{2}=x_{3}=\cdots=x_{I}=0\right)$ and another solution $\left(x_{I}=\right.$ Cap, $\left.x_{1}=x_{2}=\cdots=x_{I-1}=0\right)$ are both the best solutions of LM, but the resource allocation are different and nonequilibrium:

$$
\begin{gathered}
\text { LM: } \min f_{1}(x)=\sum_{i \in I} x_{i}, \\
\text { s.t. } \sum_{i \in I} x_{i} \leq \text { Cap, } \\
x_{i} \geq \frac{\text { Cap }}{I}, \quad \text { for } \forall x_{i} \subseteq Z, \forall i \in I,
\end{gathered}
$$

A quadratic model (QM) is proposed, which has a quadratic objective function $f_{2}(x)$ with constraints (A.2) and (A.3). The QM is convex, because $\partial f_{2}(x) / \partial x \geq 0$ and $\nabla^{2} f_{2}(x) \geq 0$. To solve the QM, the constraint (A.3) is ignored to construct a rational programming model with equations (A.2) and (A.4). Then, the constraint (A.2) is relaxed to the objective function, and a Lagrange multiplier $\lambda>0$ is proposed. A Lagrangian relaxation quadratic model (LR-QM) is constructed, which is shown in equation (A.5):

$$
\begin{gathered}
\text { QM: } \min \quad f_{2}(x)=\sum_{i \in I} x_{i}^{2} \\
\text { s.t. constraints } \quad(\text { A. } 2)-(\text { A.3 }), \\
\text { LR - QM: } \min f_{2}^{\prime}(x, \lambda)=\sum_{i \in I} x_{i}^{2}+\lambda\left(\sum_{i \in I} x_{i}-\text { Cap }\right),
\end{gathered}
$$

Setting $\partial f_{2}^{\prime} / \partial x_{i}=0$ for each flow gives

$$
x_{i}=\frac{\lambda}{2}, \quad \text { for } \forall i \in I \text {. }
$$

Further, the Karush-Kuhn-Tucker (KKT) conditions require that $\partial f_{2}^{\prime} / \partial \lambda=0$ for each link gives

$$
\sum_{i \in I} x_{i}-\text { Cap }=0
$$

Substituting for the $x_{i}$ to equation (A.7), we obtain

$$
\lambda=\frac{2 \text { Cap }}{I} \text {. }
$$

Substituting for the $\lambda$ to equation (A.6), we obtain

$$
x_{i}=\frac{\text { Cap }}{I}, \quad \text { for } \forall i \in I \text {. }
$$

Based on the above computation process, the optimal solution of QM is round (equation (A.9)) to integer, that is, $x_{i}=[\mathrm{Cap} / I]$, and the best result is $f_{2}(x)=\mathrm{Cap}^{2} / I$. That means the allocated resource in each station is same, which is an equilibrium resource allocation solution. There is a deeper reason that $x$ has a direct ratio relationship with $f_{1}(x)$ in linear function, and the growth rate is fixed. While, for the quadratic function $f_{2}(x)$, it has a growing variation rate (as shown in Figure 16). In other words, compared with the linear function, the quadratic function has higher marginal cost, when the variable is growing. Therefore, the allocated resource in each station will not be too much. That implies the effect of quadratic function, which can make the resource allocation equilibrium on spatial.

For another, on temporal allocation, the spatial allocation is ignored, and the resource allocation problem on one station can also be discussed. The process and result are similar as the above, and we can also obtain the conclusion that the quadratic function makes the resource be allocated equally on temporal. Therefore, the proving process of that on temporal is omitted. In summary, no matter on spatial or temporal, an equilibrium resource allocation solution can be obtained from the quadratic function.

To our study, if the number of controlled or left-behind passengers is large; it may cause the flow control delay or left-behind delay with huge value, which have a great negative effect on the objective. Therefore, the fluctuation of delay value tends to be small when the objective is quadratic, which can avoid the great negative effect with a large number of controlled or left-behind passengers on a few stations in a few periods. This may produce a flow control strategy with small fluctuation, which can balance the number of flow control or left-behind passengers among different stations and different periods. In summary, in Model II, the situation with large controlled passengers or left-behind passengers at a station or in a period will be avoided, and the number of controlled passengers or left-behind passengers is equilibrium among stations or flow control periods.

\section{B. The Linearization of Model I}

To test the efficiency of the proposed ISA algorithm, both the ISA algorithm and CPLEX solver will be used to solve the Model I. However, the Model I is an integer nonlinear programming model with a linear objective function, which includes two nonlinear constraints (equations (10) and (13)) and others are linear constraints. The Model I is difficult to solve by CPLEX, because the CPLEX is a linear solver and cannot recognize the nonlinear constraints. Therefore, the Model I should be linearized by nonlinear constraints.

The nonlinear constraint (10) in Model I can be replaced by the following linear constraints (B.1) and (B.2): 


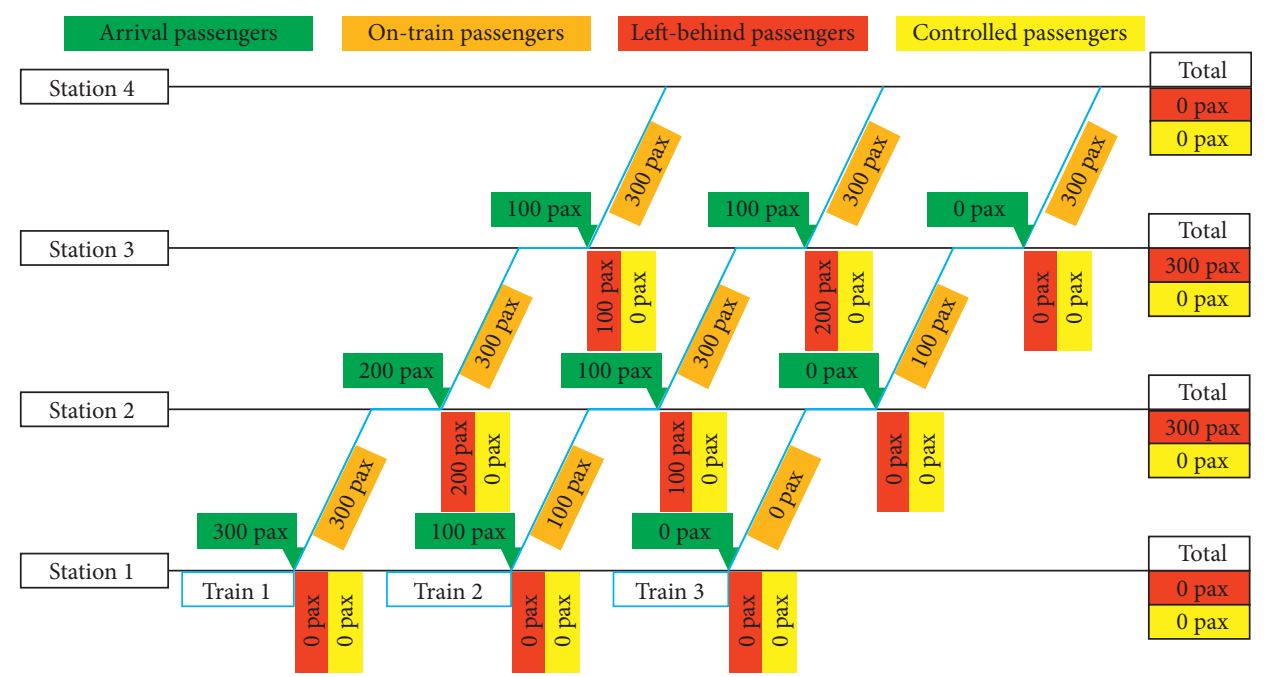

(a)

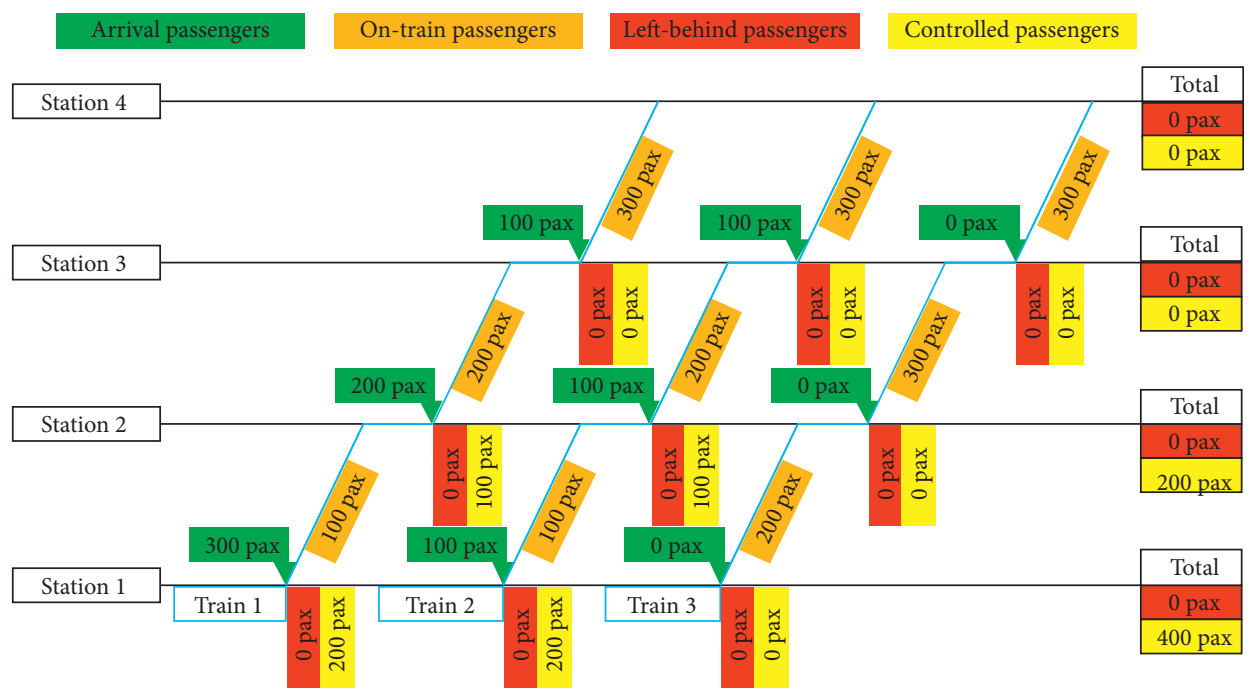

(b)

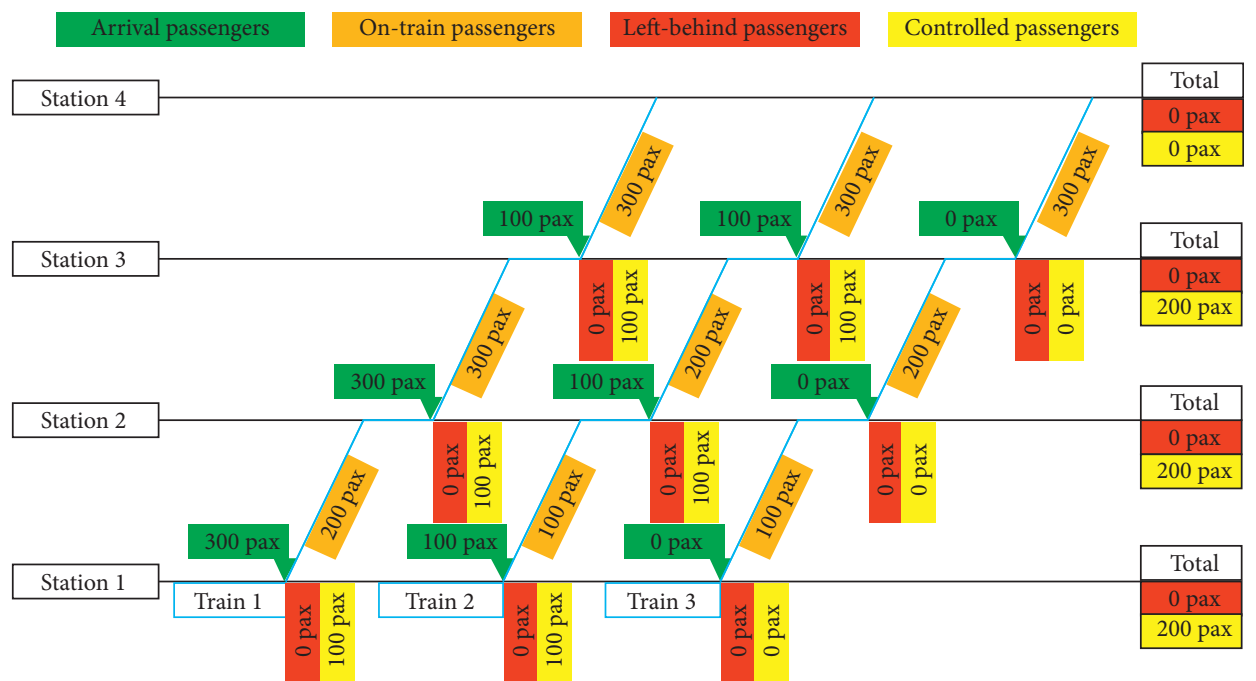

(c)

FIGURE 17: An example with different forms of flow control strategy. (a) Without any flow control strategy. (b) With nonequilibrium flow control strategy. (c) With equilibrium flow control strategy. 


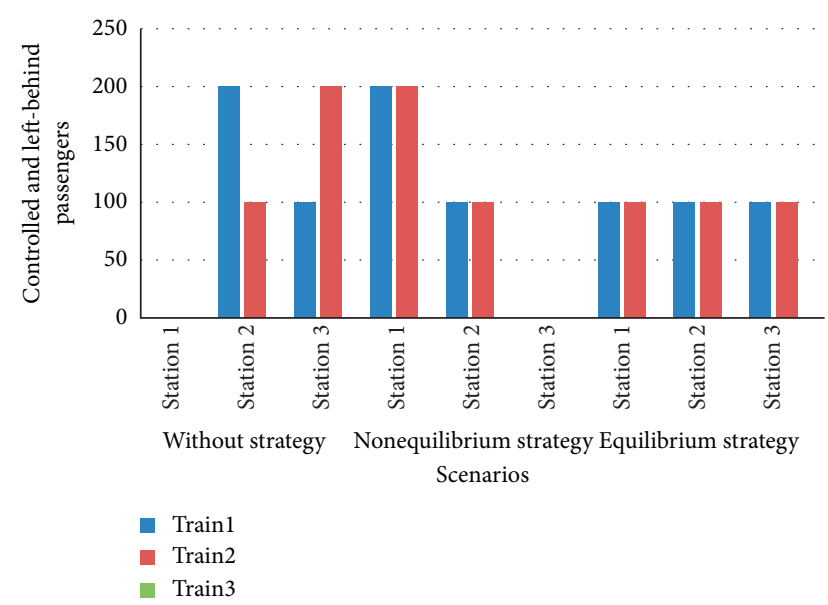

Figure 18: The comparison of controlled and left-behind passengers in different scenarios.

$$
\begin{aligned}
& \widehat{q}_{s}^{\text {control }}(t) \geq q_{s}^{\text {control }}(t)-q e_{s}^{\text {arrive }}(t), \\
& \quad \text { for } \forall s=1,2, \ldots, S-1, \forall t=2,3, \ldots, \mathrm{TE},
\end{aligned}
$$

$$
\widehat{q}_{s}^{\text {control }}(t) \geq 0, \quad \text { for } \forall s=1,2, \ldots, S-1, \forall t=2,3, \ldots, \mathrm{TE} \text {, }
$$

The nonlinear constraint (13) can be replaced by the linear constraints (B.3) and (B.4):

$$
\begin{aligned}
& q p_{s}^{\text {board }}(t) \leq \sum_{r=1}^{R} L_{s, r}(t) *\left[q p_{s}^{\text {wait }}(t-1)+q e_{s}^{\text {enter }}(t)\right] \\
& \quad \text { for } \forall s=1,2, \ldots, S-1, \forall t=2,3, \ldots, \mathrm{TE},
\end{aligned}
$$

$$
\begin{array}{r}
q p_{s}^{\text {board }}(t) \leq \sum_{r=1}^{R} L_{s, r}(t) *\left[q t_{r}^{\text {capacity }}(t-1)+q p_{s}^{\text {alight }}(t)\right] \\
\quad \text { for } \forall s=1,2, \ldots, S-1, \forall t=2,3, \ldots, \mathrm{TE} .
\end{array}
$$

With the above linearization process, the Model I can be changed to an integer nonlinear programming model, which can be solved by the CPLEX solver.

\section{An Illustrative Example about the Passenger Flow Control Strategies with Equilibrium Feature}

To explain the flow control with nonequilibrium and equilibrium strategies, an example is shown as follows. As shown in Figure 17, three trains with same 3 minutes headways run on the line containing four stations, and the capacity of each train is 300 . The number of arrival passengers at each station is shown in green grid, and their destination is station 4. Passengers will be controlled at the entrance in each period, and the length of a period is a headway of the trains. The number of passengers on the train, left behind at the platform, and controlled at the entrance is shown in orange grid, red grid, and yellow grid, respectively.

Figure 17(a) indicates the situation without any flow control strategy, and passengers follow the first-come-firstservice rule. No passengers will be controlled, and 300 passengers were left behind at station 2 and 300 passengers were left behind at station 3. Figure 17(b) follows the nonequilibrium flow control strategy. No passengers will be left behind, and 400 passengers were controlled at station 1 , and 200 passengers were controlled at station 2. A large number of passengers were controlled at the station 1 , and 200 passengers were controlled at the station 1 in one period. The result of equilibrium flow control strategy is shown in Figure 17(c). No passengers will be left behind, and 200 passengers were controlled at stations 1,2 , and 3, respectively. As shown in Figure 18, the left-behind and controlled passengers on each station in each scenario are compared. Different from the result of the above two scenarios, the number of controlled passengers with equilibrium flow control strategy were not over 100 at any station in any train, and the number of controlled passengers on each station are same for each train. Therefore, the third flow control strategy is equilibrium at successive stations and in successive periods.

In summary, the total number of controlled passengers with non-equilibrium and equilibrium flow control strategies is same, but the equilibrium flow control strategy balances the number of controlled passengers at successive stations and in successive periods. The equilibrium flow control strategy not only avoids the situation in which too many passengers were controlled at a few stations but also is easy to implement for managers in practice.

\section{Data Availability}

The (Passenger arrival rate, ratio of passengers in different OD pairs, timetable, train capacity, platform capacity, and passengers on the platform at beginning in numerical study) data used to support the findings of this study are included within the article.

\section{Conflicts of Interest}

The authors declare that they have no conflicts of interest.

\section{Acknowledgments}

This work was supported by the China Postdoctoral Science Foundation (2020M682590); the National Key Research and Development Plan (2018YFB1201402); the Fundamental Research Funds for the Central Universities (2018JBM020; 2018JBM031); the National Natural Science Foundation of 
China (71971019); the Programme of Introducing Talents of Discipline to Universities (B18004); and the TCT Funding Program (9907006511; 9907006518).

\section{References}

[1] S. Li, M. M. Dessouky, Z. Gao, and Z. Gao, "Joint optimal train regulation and passenger flow control strategy for high-frequency metro lines," Transportation Research Part B: Methodological, vol. 99, pp. 113-137, 2017.

[2] X.-Y. Xu, J. Liu, H.-Y. Li, M. Jiang, and W. Talley, "Capacityoriented passenger flow control under uncertain demand: algorithm development and real-world case study," Transportation Research Part E: Logistics and Transportation Review, vol. 87, pp. 130-148, 2016.

[3] X. Xu, H. Li, J. Liu, B. Ran, and L. Qin, "Passenger flow control with multi-station coordination in subway networks: algorithm development and real-world case study," Transportmetrica B: Transport Dynamics, vol. 7, no. 1, pp. 446-472, 2019.

[4] H. Niu and X. Zhou, "Optimizing urban rail timetable under time-dependent demand and oversaturated conditions," Transportation Research Part C: Emerging Technologies, vol. 36, no. 11, pp. 212-230, 2013.

[5] E. Barrena, D. Canca, L. C. Coelho, and G. Laporte, "Singleline rail rapid transit timetabling under dynamic passenger demand," Transportation Research Part B: Methodological, vol. 70, pp. 134-150, 2014.

[6] L. Sun, J. G. Jin, D.-H. Lee, K. W. Axhausen, and A. Erath, "Demand-driven timetable design for metro services," Transportation Research Part C: Emerging Technologies, vol. 46, pp. 284-299, 2014.

[7] F. Delgado, J. C. Muñoz, R. Giesen, and A. Cipriano, "Realtime control of buses in a transit corridor based on vehicle holding and boarding limits," Transportation Research Record: Journal of the Transportation Research Board, vol. 2090, no. 1, pp. 59-67, 2009.

[8] F. Delgado, J. C. Muñoz, and R. Giesen, "How much can holding and/or limiting boarding improve transit performance?" Transportation Research Part B: Methodological, vol. 46, no. 9, pp. 1202-1217, 2012.

[9] O. J. Ibarra-Rojas, F. Delgado, R. Giesen, and J. C. Muñoz, "Planning, operation, and control of bus transport systems: a literature review," Transportation Research Part B: Methodological, vol. 77, pp. 38-75, 2015.

[10] G. E. Sánchez-Martínez, H. N. Koutsopoulos, and N. H. M. Wilson, "Real-time holding control for high-frequency transit with dynamics," Transportation Research Part B: Methodological, vol. 83, pp. 1-19, 2016.

[11] S. Baee, F. Eshghi, S. M. Hashemi, and R. Moienfar, "Passenger boarding/alighting management in urban rail transportation," in Proceedings of the Joint Rail Conference, pp. 17-19, Philadelphia, PA, USA, April 2012.

[12] W. Li and J. Zhou, "The optimize management of passenger organization in transfer station based on dynamic passenger flow analysis," Procedia-Social and Behavioral Sciences, vol. 96, pp. 1322-1328, 2013.

[13] S. Sebastián and R. Fernandez, "Pedestrian traffic management of boarding and alighting in metro stations," Transportation Research Part C: Emerging Technologies, vol. 53, pp. 76-92, 2015.

[14] Z. He, Y. Chen, L. Sun, J. Zhong, and Y. Zhu, "Identifying station-link correlation for target passenger flow control in subway network," in Proceedings of the 21st International
Conference on Intelligent Transportation Systems (ITSC), pp. 84-89, Maui, HI, USA, November 2018.

[15] M. Jiang, H.-y. Li, X.-y. Xu, S.-p. Xu, and J.-r. Miao, "Metro passenger flow control with station-to-station cooperation based on stop-skipping and boarding limiting," Journal of Central South University, vol. 24, no. 1, pp. 236-244, 2017.

[16] Z. Jiang, W. Fan, W. Liu, B. Zhu, and J. Gu, "Reinforcement learning approach for coordinated passenger inflow control of urban rail transit in peak hours," Transportation Research Part C: Emerging Technologies, vol. 88, pp. 1-16, 2018.

[17] Z. Jiang, J. Gu, W. Fan, W. Liu, and B. Zhu, "Q-learning approach to coordinated optimization of passenger inflow control with train skip-stopping on a urban rail transit line," Computers \& Industrial Engineering, vol. 127, pp. 1131-1142, 2019.

[18] J. Shi, L. Yang, J. Yang, and Z. Gao, "Service-oriented train timetabling with collaborative passenger flow control on an oversaturated metro line: an integer linear optimization approach," Transportation Research Part B: Methodological, vol. 110, pp. 26-59, 2018.

[19] R. Liu, S. Li, and L. Yang, "Collaborative optimization for metro train scheduling and train connections combined with passenger flow control strategy," Omega, vol. 90, Article ID 101990, 2018.

[20] J. Shi, L. Yang, J. Yang, F. Zhou, and Z. Gao, "Cooperative passenger flow control in an oversaturated metro network with operational risk thresholds," Transportation Research Part C: Emerging Technologies, vol. 107, pp. 301-336, 2019.

[21] F. Yuan, H. Sun, L. Kang, and J. Wu, "Passenger flow control strategies for urban rail transit networks," Applied Mathematical Modelling, vol. 82, pp. 168-188, 2020.

[22] H. Xue, L. Jia, and J. Guo, "Adaptive multilevel collaborative passenger flow control in peak hours for a subway line," Advances in Mathematical Physics, vol. 2020, Article ID 3862157, 16 pages, 2020.

[23] Z. Huang, R. Xu, W. Fan, F. Zhou, and W. Liu, "Serviceoriented load balancing approach to alleviating peak-hour congestion in a metro network based on multi-path accessibility," Sustainability, vol. 11, no. 5, p. 1293, 2019.

[24] S. Hao, R. Song, S. He, and Z. Lan, "Train regulation combined with passenger control model based on approximate dynamic programming," Symmetry, vol. 11, no. 3, p. 303, 2019.

[25] Ö. Yalçınkaya and G. M. Bayhan, "Modelling and optimization of average travel time for a metro line by simulation and response surface methodology," European Journal of Operational Research, vol. 196, no. 1, pp. 225-233, 2009.

[26] I. H. Osman, "Metastrategy simulated annealing and tabu search algorithms for the vehicle routing problem," Annals of Operations Research, vol. 41, no. 4, pp. 421-451, 1993.

[27] S. Kirkpatrick, C. D. Gelatt, and M. P. Vecchi, "Optimization by Simulated Annealing," Readings in Computer Vision, pp. 606-615, Elsevier, Amsterdam, Netherlands, 1987.

[28] E. Aarts, "Simulated annealing and Boltzmann machines," Handbook of Brain Theory \& Neural Networks, MIT Press, Cambridge, MA, USA, 1989.

[29] R. Paul, D. Alan, W. Mark et al., "Transit capacity and quality of service manual," Transit Cooperative Research Program (TCRP), Vol. 165, Transportation Research Board, Washington, DC, USA, 3rd edition, 2013. 